\title{
Spatiotemporal dimensionality and Time-Space characterization of multitemporal imagery
}

\author{
Christopher Small * \\ Lamont Doherty Earth Observatory, Columbia University Palisades, NY 10964, USA
}

\section{A R T I C L E I N F O}

\section{Article history:}

Received 28 August 2011

Received in revised form 26 May 2012

Accepted 30 May 2012

Available online $\mathrm{xxxx}$

\section{Keywords:}

Spatiotemporal

Spatial

Temporal

Dimensionality

Principal component

Empirical orthogonal function analysis

EOF

PCA

Temporal mixture model

Spectral mixture model

Deterministic

Stochastic

\begin{abstract}
A B S T R A C T
Spatiotemporal dimensionality refers to the continuum of spatial and temporal patterns in an image time series. Time-Space characterization refers to a way of representing this continuum of patterns as combinations of spatial and temporal constituents - with a minimum of assumptions about the forms of the patterns. Patterns can be related to processes through modeling. By combining characterization and modeling, two complementary analytical tools can be used together so that each resolves a key limitation of the other. This study describes a straightforward extension of Principal Component Analysis and Spectral Mixture Analysis to multitemporal imagery and illustrates how characterization of the dimensionality and eigenstructure of the data can inform modeling of the processes represented in the data. The relationships among spatiotemporal processes can be represented as combinations of temporal endmembers in a temporal feature space where the dimensions represent different components of the temporal patterns present in the data. The topology of the feature space and the processes being modeled together inform the selection of temporal endmembers and the structure of the model chosen to represent the processes. The dimensionality revealed by the characterization can also provide a partial solution to the problem of endmember variability. The characterization and modeling process is illustrated with the vegetation phenology of the Ganges-Brahmaputra delta using a MODIS vegetation index time series. Additional applications and limitations of Time-Space characterization and mixture modeling are further illustrated by comparing the eigenstructures and temporal feature spaces of Landsat vegetation fraction and DMSP-OLS night light time series.
\end{abstract}

(C) 2012 Published by Elsevier Inc.

\section{Introduction}

Over the past $30+$ years, broad interest in Earth surface processes has led to a variety of approaches to quantify remotely sensed change. The diversity of approaches has resulted in multiple review papers which have developed categorizations of the different approaches for change detection and their relative strengths and weaknesses. (Coppin \& Bauer, 1996; Coppin et al., 2004; Lu et al., 2004). However, most of this work has focused on discrete change detection. The more general problem of quantifying continuous spatial and temporal changes has received less attention. The relatively recent availability of large volumes of multitemporal imagery at hectometer (e.g. MODIS) to decameter (e.g. Landsat) resolutions now makes it more feasible to consider the related problem of spatiotemporal (ST) analysis. Spatiotemporal analysis can be considered distinct from discrete change detection in the sense that it simultaneously quantifies both temporal patterns and their spatial distribution. Proposed approaches for spatiotemporal analysis include logistic function fitting (Zhang et al., 2006), wavelet decomposition (Galford et al., 2008), Independent Component Analysis

\footnotetext{
* Tel.: +1 3475354274 .

E-mail address: small@LDEO.columbia.edu.
}

(Ozdogan, 2010), trend and change decomposition (Verbesselt et al., 2010), and temporal mixture modeling (Lobell \& Asner, 2004; Piwowar et al., 1998; deBeurs \& Henebry, 2006). Most of these approaches involve some assumptions about the temporal patterns in the data. However, with image time series the "Curse of Dimensionality" (Bellman, 1957) often arises in the challenge of determining what spatial and temporal patterns are actually present in the data and what method is best suited to quantifying them. Characterization of high dimensional data in terms of its dimensionality provides a way to represent the high dimensional information content of multitemporal imagery while managing the challenges modeling the underlying processes.

Characterization of data can be considered the complement of modeling. Modeling involves the representation of observations or processes with a conceptual or mathematical simplification. (Gershenfeld, 1999). Forward modeling simulates a process given a set of parameters while model inversion seeks optimal estimates of model parameters or structure corresponding to a set of observations (Parker, 1994). Modeling generally involves assumptions about the functional form of the processes represented by the data. The implicit assumptions are usually that the functional form of the processes is known a priori and that the model parameter estimates convey something about the processes. Forward modeling of atmospheric effects with a radiative transfer model is 
an example of deterministic modeling (e.g. (Tanre et al., 1979)). Estimation of phenological parameters by fitting logistic functions to time series of vegetation indices is an example of model inversion (e.g. (Zhang et al., 2006)). In contrast, characterization of data can take the form of an exploratory data analysis and thereby makes less rigid assumptions about processes and their representation in the data. (Tukey, 1977). The assumption of a specific functional form is not required for characterization. Identification of uncorrelated modes of variability using Principal Component transform is a common example of data characterization. (von Storch \& Zwiers, 1999) The analogy to supervised and unsupervised classification used by (Verbesselt et al., 2010) to describe change detection methods can also be applied to spatiotemporal analysis. Data characterization can be considered analogous to unsupervised classification in that it identifies patterns in data with few assumptions from the analyst. Data modeling is analogous to supervised classification in that the result is strongly dependent on assumptions and input from the analyst. Both modeling and characterization have strengths and limitations. When used together they can complement and inform each other.

The idea of spatiotemporal dimensionality provides a basis for characterization of multitemporal imagery and the development of spatiotemporal models. In the context of this study spatiotemporal dimensionality refers to the structure of the continuum of spatial and temporal patterns present in an image time series. The spatiotemporal dimensionality of an image time series is related to the number and combination of processes that can be distinguished at different geographic locations through time. Before using data to quantify or infer spatiotemporal processes, it is important to know if and how these processes are represented in the data. Characterization of multitemporal imagery can provide insights into how different processes are represented by the spatial and temporal sampling of the imagery. The purpose of characterization is not only to identify specific features but also to determine what can and cannot be distinguished in the data - with a minimum of assumptions. While characterization can inform any of the modeling approaches referenced previously, the continuous representation of spectral mixture models is particularly well-suited to characterization in terms of Principal Components.

This study presents an approach to characterizing and modeling spatiotemporal processes in multitemporal imagery. The combined approach of Time-Space characterization and spatiotemporal modeling is developed using Empirical Orthogonal Function analysis and Temporal Mixture Models. The approach follows a strategy developed for spectral mixture analysis (Adams et al., 1986) but addresses some important differences between spectral and spatiotemporal dimensionality and the physical processes they represent. The Principal Component (PC) transformation and resulting Empirical Orthogonal Functions (EOFs) provide a tool for representing the spatiotemporal dimensionality of an image time series in the form of uncorrelated temporal patterns (EOFs) and their spatial distributions (PCs). The dimensionality and structure of the temporal feature space reveals the dominant temporal patterns and the relationships among them. The Temporal Mixture Model provides a tool for modeling and mapping spatial relationships among the temporal patterns as processes. When used together, each tool resolves a key limitation of the other. The characterization of the dimensionality informs the design of the mixture model while the use of the mixture model eliminates the difficulty of direct interpretation of the individual EOFs. The spatiotemporal (ST) dimensionality determined from the EOF analysis also provides a potential solution to a principal challenge of mixture modeling: endmember selection and variability. This is achieved through a separation of high and low order variance as indicated by results of the EOF analysis.

This study illustrates the combined use of characterization and modeling with a combination of theory and application. The intention is to illustrate both strengths and limitations of the approach by comparison of examples. The common theoretical basis and mathematical similarities of EOF analysis and linear mixture modeling are discussed first. This is followed by a worked example of characterization of the relatively well-posed problem of phenology mapping with a time series of MODIS-derived vegetation index images. This example is followed by a brief comparison of two more challenging examples intended to highlight some effects of differences in dimensionality and eigenstructure. The use of three contrasting examples illustrates both the generality of the characterization approach and the diversity of spatiotemporal structure of different image time series. It also illustrates some limitations of the use of purely statistical transformations in the representation of high dimensional data.

\section{Principal components and empirical orthogonal functions}

PC transformations are commonly used to represent uncorrelated modes of variance in high dimensional data. Different types of PC transform are used to reduce the dimensionality of multispectral imagery (e.g. (Green et al., 1988; Lee et al., 1990; Singh \& Harrison, 1985)) and to represent the topology of spectral feature spaces (Adams et al., 1986; Crist \& Cicone, 1984; Johnson et al., 1985; Kauth \& Thomas, 1976; Smith et al., 1985). Because spectral bands are often correlated, PC transforms provide an efficient low dimensional projection of the uncorrelated components of the spectral feature space. The same property applies to temporal dimensions. PC transforms have also been used to represent uncorrelated patterns in multitemporal imagery (Richards, 1984) (Eastman \& Fulk, 1993; Townshend et al., 1985) and for change detection (Byrne et al., 1980; Fung \& LeDrew, 1987). In meteorology and oceanography the PC transformation provides the basis of Empirical Orthogonal Function analysis; a standard tool for analysis of spatiotemporal patterns and processes. (see (Bretherton et al., 1992; Preisendorfer, 1988; von Storch \& Zwiers, 1999) for overviews).

The PC transform provides a very convenient tool for identification of spatiotemporal patterns. By rotating the coordinate system to align with orthogonal dimensions of uncorrelated variance, any location-specific pixel time series $P_{x t}$ contained in an $\mathrm{N}$ image time series can be represented as a linear combination of temporal patterns, $F$, and their location-specific components, $C$, as:

$P_{x t}=\sum_{i=1}^{N} C_{i x} F_{i t}$

where $C_{i x}$ is the spatial Principal Component $(\mathrm{PC})$ and $F_{i t}$ is the corresponding temporal Empirical Orthogonal Function (EOF) and $i$ is the dimension. The EOFs are the eigenvectors of the covariance matrix that represent uncorrelated temporal patterns of variability within the data. The PCs are the corresponding spatial weights that represent the relative contribution of each temporal EOF to the corresponding pixel time series $P_{x t}$ at each location $x$. The relative contribution of each EOF to the total spatiotemporal variance is given by the eigenvalues of the covariance matrix. $N$ is the number of discrete dimensions represented by the data; which may be greater, or less, than the true physical dimensionality of the process(es) imaged. Principal Components are uncorrelated but not necessarily independent - unless the data are jointly normally distributed. In systems where the same deterministic processes are manifest at many locations, but stochastic processes are uncorrelated, the variance of the spatiotemporal structure of the deterministic processes can be represented in the low order PC/EOF dimensions while the stochastic variance is represented in the higher order dimensions (Preisendorfer, 1988). When a clear distinction can be made, this can provide a statistical basis for separation of deterministic and stochastic components of an image time series. However, the 
transformation is purely statistical so there is no guarantee that the separation is physically meaningful.

In oceanography and meteorology the PC transformation is often used to characterize dynamically important modes of spatiotemporal variance associated with physical processes. With noisy observations the implicit assumption is that some number, $\mathrm{D}(\leq \mathrm{N})$, of the low order dimensions correspond to pervasive deterministic processes and that the higher order dimensions represent stochastic variance $\varepsilon$. This allows an observed pixel time series to be represented as a sum of deterministic and stochastic components:

$P_{x t}=\sum_{i=1}^{D} C_{i x} F_{i t}+\epsilon$

where the weighted sum of low order dimensions, $C_{i x} F_{i t}$, represents the deterministic component and the residual, $\varepsilon$, represents the stochastic variance. With ocean and atmospheric circulation this may be a valid assumption, however the distinction between deterministic and stochastic land surface processes is not necessarily scale dependent so this categorization may not be valid for all cases. For this reason, these components will generally be referred to as simply low order dimensions and the higher order residual. In conventional usage, EOFs are usually spatial patterns intended to represent spatially continuous modes of variability of physical processes while the PCs are usually the weights representing the temporal contribution of the corresponding spatial pattern (Preisendorfer, 1988; von Storch, 1999). In this study the convention is reversed and the EOFs represent temporal patterns. The reason for reversing the convention will be explained in the discussion of the examples.

\subsection{The challenge of EOF analysis}

The PC transformation produces statistically uncorrelated dimensions of spatiotemporal variance but does not guarantee, or even imply, physical significance. While the transformation may separate physically distinct processes, it is purely statistical - not dynamical. Individual EOFs may be physically meaningful, but they are not required to be. Because each EOF is combined with all other EOFs (that have non-zero PCs) they generally interfere both constructively and destructively. This leads to a primary challenge of EOF analysis: interpretation of the EOFs. A single spatiotemporal process can be manifest in more than one EOF and multiple processes may contribute to a single EOF. As a result, approaches have been developed for "rotating" or recombining EOFs to produce new patterns that may be easier to interpret (see von Storch \& Zwiers, 1999). In the PC-based multitemporal studies discussed above the interpretations focus on the individual low order EOFs (also referred to as loadings) and the spatial patterns of the corresponding PCs. A partial solution to the challenge of EOF interpretation is provided by temporal mixture models.

\section{Temporal mixture models}

Temporal mixture models are the temporal analogue to spectral mixture models (Adams et al., 1986; Gillespie et al., 1990; Smith et al., 1990) commonly used to represent spectrally mixed pixels. Like the PC transform, a temporal mixture model represents a pixel time series as a combination of temporal patterns (endmembers) and residual variance. Each location-specific (x) pixel $P_{x t}$ in an $\mathrm{N}$ image time series can be represented as a linear combination of $\mathrm{D}^{\prime}$ temporal endmembers, $E_{i t}$, and a residual component, $\varepsilon$, as:

$P_{x t}=\sum_{i=1}^{D^{\prime}} f_{i x} E_{i t}+\epsilon$ where the pixel-specific fractions $f_{i x}$ may represent either the areal fraction of the pixel exhibiting the temporal pattern of the corresponding endmember or, more generally, the Euclidian proximity of that pixel to the corresponding endmember in the temporal feature space. The result is a set of fraction (more generally proximity) maps representing the spatial distribution of different endmember abundances (or similarities). Dynamically, the temporal endmembers, $E_{i t}$, can represent distinct processes and $\varepsilon$ represents the unmodeled residual component of the observations. The model is generally parsimonious if the modeled dimensionality, $\mathrm{D}^{\prime}$, is assumed less than or equal to the true dimensionality. Given an observed pixel time series $P_{x t}$ with sufficient temporal sampling, and knowledge of a relatively small number of endmembers, $E_{i t}$, the linear mixture model, $\mathrm{P}=\mathrm{f} \mathrm{E}+\varepsilon$, can be inverted to yield fraction estimates of each endmember for each pixel (Boardman, 1989; Settle \& Drake, 1993). This leads to the primary challenge of mixture modeling: selection of endmembers.

\subsection{The challenge of mixture modeling}

As in spectral mixture modeling, the number, $\mathrm{D}^{\prime}$, and choice of endmembers $E_{i}$ chosen to span the mixing space determines the accuracy and validity of the results. Selection of EMs therefore involves an implicit assumption about the spatiotemporal dimensionality of the processes represented in the imagery. A variety of different approaches have been used to select temporal endmembers. Studies of vegetation are able to make use of higher spatial resolution imagery to identify specific types of vegetation as phenological endmembers. In a study of agricultural land cover (Quarmby et al., 1992) used a classified SPOT HRV imagery to derive training endmembers for a 4 endmember model based on rice, maize, cotton and wheat. In a more recent study of cropland distributions using Quality Assessment-constrained MODIS reflectance data (Lobell \& Asner, 2004) developed a probabilistic temporal unmixing procedure in which Landsat classifications inform the selection of suites of temporal endmembers. These endmember suites are then used as the basis of a Monte Carlo procedure that repeatedly samples each endmember suite to produce a distribution of mixture models to reduce sensitivity to endmember variability. The alternative to using auxiliary information to identify endmembers a priori is to derive the endmembers directly from the multitemporal imagery. In a study of Arctic sea ice dynamics (Piwowar et al., 1998) used RMS misfit to a simple two endmember model to isolate two additional temporal endmembers and compared the results of different endmember combinations. The authors comment in the discussion that PC analysis could also be used to assist in endmember selection. In a later study of AVHRR-derived vegetation indices (Piwowar et al., 2006) use an iterative reprojection procedure to automate selection of candidate endmembers on the basis of their brightness and orthogonality then select four that represent processes of interest for their study. Taking a different approach, (deBeurs \& Henebry, 2006) construct mixture models of phenological endmembers. The endmembers are represented by parameterizing five different spectral-temporal characteristics of vegetation phenology as a function of accumulated growing degree days (AGDD). This type of parameterization represents an important advance in temporal mixture modeling because it represents the general process as a combination of more fundamental processes defined in terms of parameters of analytic functions defined on a transformed dimension (AGDD) rather than as simple time series of one specific measurement.

As with spectral mixture models, there appears to be no consensus on how best to select temporal endmembers. The studies above use a variety of approaches, each appropriate to the problem at hand, but none of the approaches addresses the underlying problem of identification of similar spatiotemporal patterns in a general context. Whatever selection method is used to identify the EMs, the combination of EMs must be consistent with the topology of the temporal feature 
space. Even if EMs are chosen apriori it is still important to understand their relationship to the data to verify the consistency of the model with the data. This underscores the importance of characterization. Characterization can reveal the diversity of temporal endmembers (tEMs) and their relative contributions to the spatiotemporal processes represented by the data.

\subsection{Complementarity of EOF analysis and mixture modeling}

The challenges of EOF interpretation and endmember selection can potentially be resolved simultaneously by considering process-specific $(p)$ endmembers, $E_{p}$, not from individual time series containing stochastic variance but from their common low order components:

$E_{p t}=\sum_{i=1}^{D} C_{i p} F_{i t}$

made up of linear combinations of temporal EOFs, $\left(F_{i t}\right)$ and their corresponding spatial PCs, $\left(C_{i p}\right)$ derived from a subspace of D dimensions representing the low order processes of interest - but excluding the variance, $\varepsilon$ in the higher order dimensions of the transformed space. Note that this is the same D used in Eq. (2). The number of relevant dimensions is determined by the eigenstructure of the covariance matrix while the choice of endmembers can be informed by the topology of the temporal feature space of the low order PCs.

\subsection{A strategy for spatiotemporal characterization and modeling}

A prescription for Time-Space characterization involves 1) assessment of the spatiotemporal dimensionality, $\left(\mathrm{D}^{\prime}\right)$ from the eigenstructure of the data, 2) identification of the process-relevant subspace of the temporal feature space, 3 ) selection of endmembers, 4) estimation of fractions and 5) validation of the model, 6) refinement of the model (if necessary) and 7) interpretation of the results. The eigenstructure of the image time series' covariance matrix provides the EOFs and their relative contributions to the spatiotemporal variance. The topology of the temporal feature space of the low order PCs provides a basis for selecting the linear combinations of EOFs representing specific processes of interest. The dimensionality of the space is indicated by the temporal structure of the EOFs and the spatial structure of the PCs and the relative contribution of each dimension to the total spatiotemporal variance of the image time series. Steps 1 through 4 of the process are illustrated here with a progression of three example analyses. Validation, refinement and interpretation of the three examples are presented in separate, location-specific studies. The studies range from a well-posed example where the relevant ST processes are oversampled in time and space to an ill-posed example where a simple linear mixture model does not accurately represent the processes. An intermediate example illustrates the case where temporal sampling introduces aliasing that can be accommodated by the choice of an appropriate mixture model.

\section{Well-posed problem: inter-annual vegetation phenology from MODIS-derived vegetation indices}

The Ganges-Brahmaputra delta (GBD) provides a diverse juxtaposition of natural and anthropogenic processes encompassing a range of spatiotemporal structure. The diversity of vegetation in these natural and human-dominated environments responds to the annual flooding events that result from the convergence of dual melt water pulses from the Tibetan Plateau with the arrival of the summer monsoon. All of these processes exert powerful influences on the phenology of both indigenous vegetation and the diversity of agriculture occurring on the delta. The spatial patterns and magnitudes of temporal variability are represented in the temporal moment composite in Fig. 1. This composite uses low order moments of the temporal distribution of MODIS Enhanced Vegetation Index (EVI)(Huete et al., 2002) values to show a spatial view of the temporal variability of vegetation on the delta. EVI is calculated for 243 composites at 16 day temporal and $250 \mathrm{~m}$ spatial resolution over the past decade. The resulting temporal moment composite shows spatial variations in vegetation abundance (mean EVI) as well as two measures of temporal variability (Standard Deviation and Mean Absolute Deviation). The greater sensitivity of the Standard Deviation to extreme values highlights areas with more frequent cloud contamination. While the temporal moment composite shows the spatial distribution and temporal variability of the vegetation, it reveals nothing of the vegetation phenology.

\subsection{Eigenstructure and characterization}

The eigenstructure of the covariance matrix of MODIS EVI for the GBD shows a relatively clear distinction between periodic and aperiodic modes of temporal variability. The eigenvalue spectrum attributes $81 \%$ of the spatiotemporal variance to the first three dimensions and shows a clear distinction between the eight largest eigenvalues and the continuum of smaller eigenvalues (Fig. 2). Qualitatively, the eigenvalues reveal a relatively sharp discontinuity between the 8 low order dimensions with rapidly diminishing variance (even in $\log _{10}$ space) and the continuum of slowly diminishing eigenvalues representing the remaining 235 dimensions accounting for the remaining $19 \%$ of variance.

The temporal structure of the EOFs also shows a pronounced difference between the 8 low order dimensions and the remaining 235 . The 8 low order EOFs have relatively smooth annual and biannual variations but higher order EOFs are characterized by alternating narrow bands of higher frequency (16-48 day) temporal variance separated by broader bands of low temporal variance (Fig. 2 insets). The period of the banding is annual but the phase of the high frequency bands shifts seasonally from low to high order EOFs. Dynamically, this progressive phase shift of high frequency variance represents the contrast between the high day to day variability in cloud cover during the summer monsoon and much lower variance associated with the winter dry season. The abrupt break in EOF structure suggests a dynamical distinction between more deterministic periodic components driven by cycles of monsoon and flooding and the more stochastic spatiotemporal variance associated with cloud contamination.

The distinction between the high and low order EOFs can be quantified by a comparison of their time domain and frequency domain depictions. Fig. 3 shows the first 12 EOFs as temporal vectors with power spectral density estimates for each. Power spectral density, estimated using both Welch's Modified Periodigram and Thompson's Multitaper methods, (Percival \& Walden, 1993) characterizes the temporal variance structure of the EOFs in terms of dominant periodicities (peaks) and aperiodic background variance. Both the time and frequency domain depictions of the EOFs show a clear distinction between the 8 low order modes and the continuum of higher order modes. As expected, the first EOF corresponding to the temporal mean EVI is flat with effectively no variance at subdecadal periods. This is reflected in the extremely red power spectrum with significant variance only at the longest temporal periods. The second and third EOFs are relatively monochromatic with peaks at annual and biannual periods (respectively). The remaining five low order modes are more polychromatic with combinations of annual and subannual periods. EOFs 7 and 8 are distinct in that they have significant variance at both subannual and longer inter-annual periods. This is apparent in both the time domain EOFs and the peaks of their corresponding power spectra. Note that the inter-annual trends of these two EOFs tend to mirror each other. This illustrates the degeneracy that 


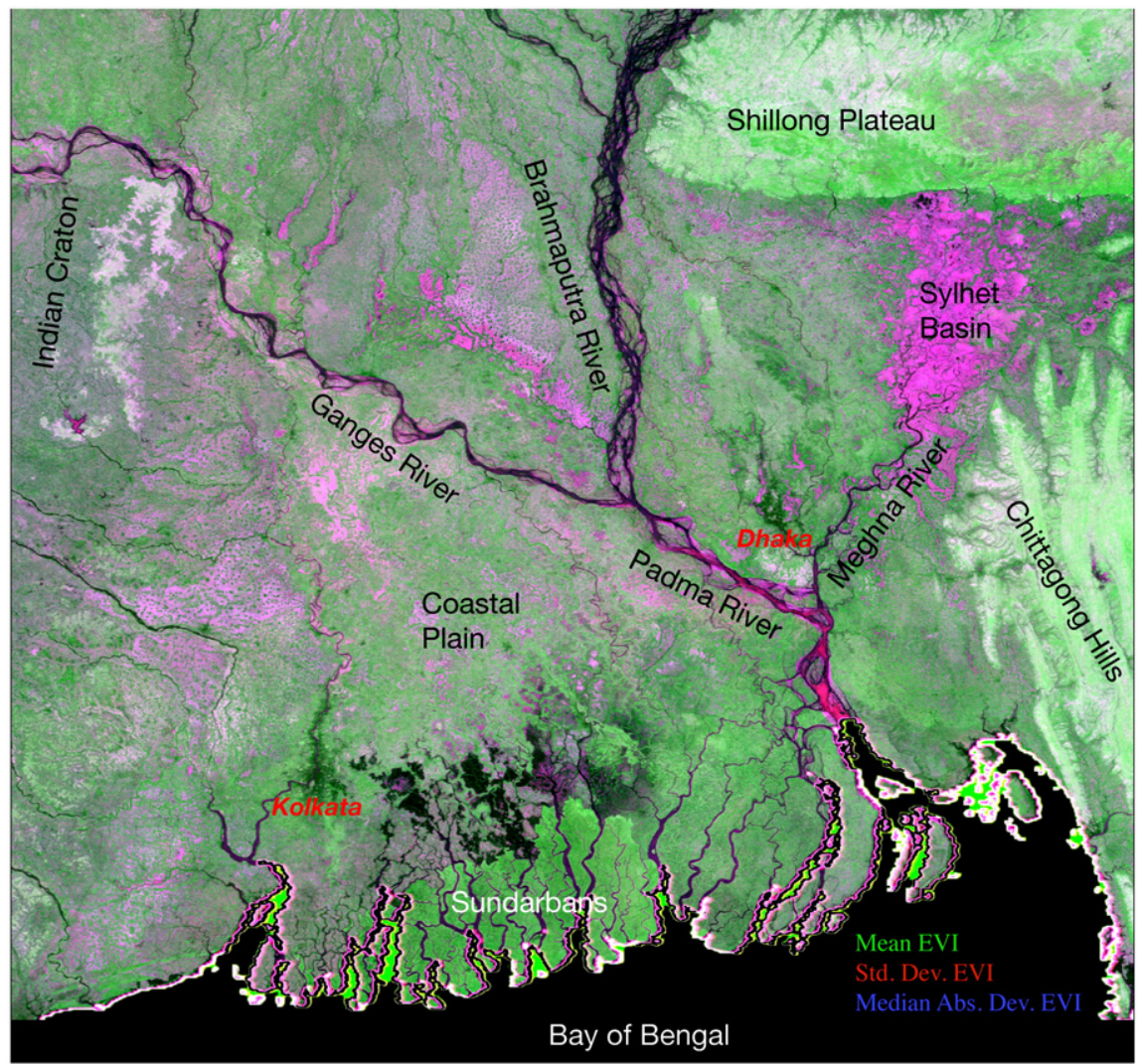

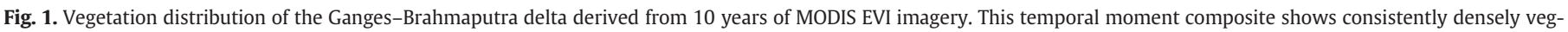

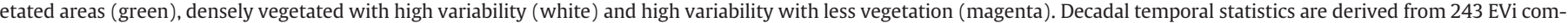
posites with $250 \mathrm{~m}$ spatial and 16 day temporal resolution.

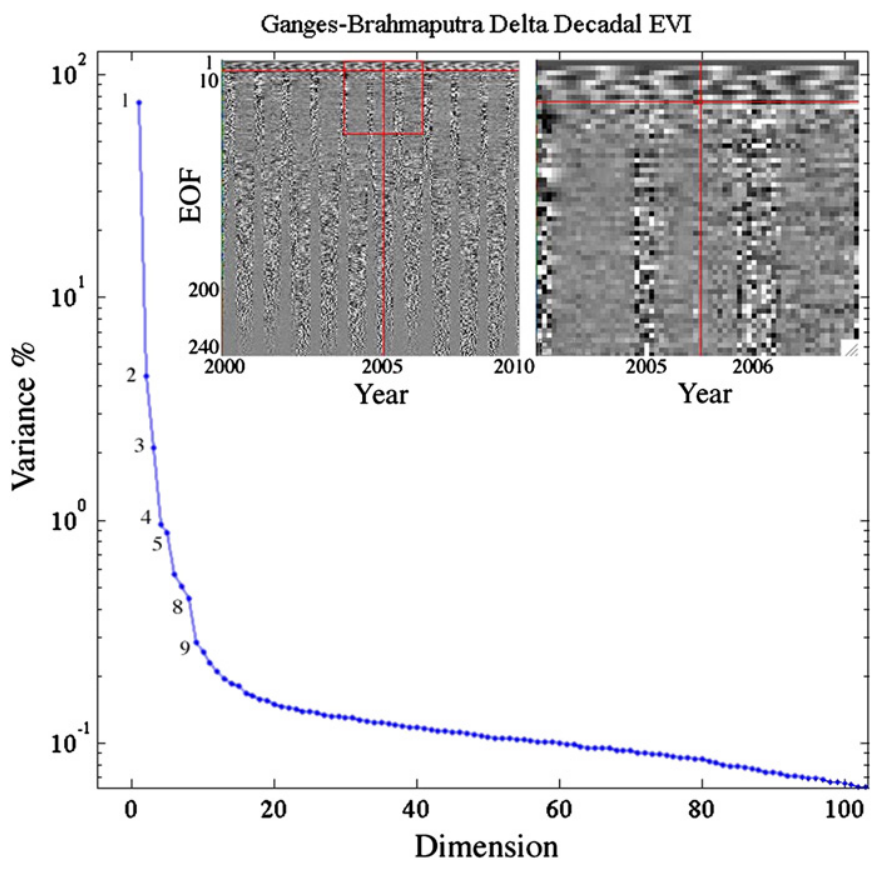

Fig. 2. Eigenstructure of the Ganges-Brahmaputra EVI image time series. The eigenvalue spectrum attributes $81 \%$ of variance to the first three dimentions and shows a clear distinction between the eight largest eigenvalues and the continuum of smller eigenvalues. The inset matrix of 243 full EOFs (1) and the zoom (r) also show a conspicuous distinction between the eight low order EOFs above the red horizontal and the continuum below. Note the annual oscillation of the high frequency structure between the low and high order EOFs. Thevariation along the red vertical progresses from low to high variance with increasing dimention. complicates interpretation of individual EOFs. In contrast, the higher order $(>8)$ aperiodic EOFs are quite different in their nearly white spectra lacking any distinct peaks at subdecadal periods.

The dimensionality seen in the eigenvalue spectrum and EOF structure is also manifest in the spatial structure of the EOF weightings given by the PCs. The spatial scale of the variance structure can be quantified with the spatial autocorrelation of the PCs. Moran's I autocorrelation, computed for lag distances of 1 to 30 times the $250 \mathrm{~m}$ pixel size for each PC, is shown along with the first 16 spatial PCs in Fig. 4. Larger lag distances quantify the spatial variance associated with coarser scale structure while the smaller lags are sensitive to variance at finer scales. For all PCs the autocorrelation drops off monotonically with increasing lag distance before reaching background levels at lag scales of $\sim 8$ to 12 pixels (2-3 km). The autocorrelation of PCs 1,7 and 8 are distinct from the others in their steeper descent to lower background levels over larger lag distances but the autocorrelations of all the other PCs follow a more continuous progression with increasing dimension. While the drop in autocorrelation with lag distance characterizes the spatial scale of variance in the PCs, the drop in autocorrelation with increasing PC dimension at a given lag distance is related to the spatiotemporal dimensionality of the data for variance at specific spatial scales (associated with a specific lag distance). The autocorrelation at a lag distance of 1 pixel (250 m) diminishes with increasing PC over the first 20 PCs before reaching a background level of correlation. In contrast, the autocorrelation at a lag distance of 30 pixels diminishes much more rapidly suggesting that only 8 to 10 dimensions represent most of the spatial structure at this coarser spatial scale. The drop in autocorrelation with lag distance and PC number quantifies what is visually apparent in the spatial structure of the PCs; most of the spatially coherent variance is associated with the 8 to 12 low order dimensions of the EVI image time series. The autocorrelation function is a spatially 

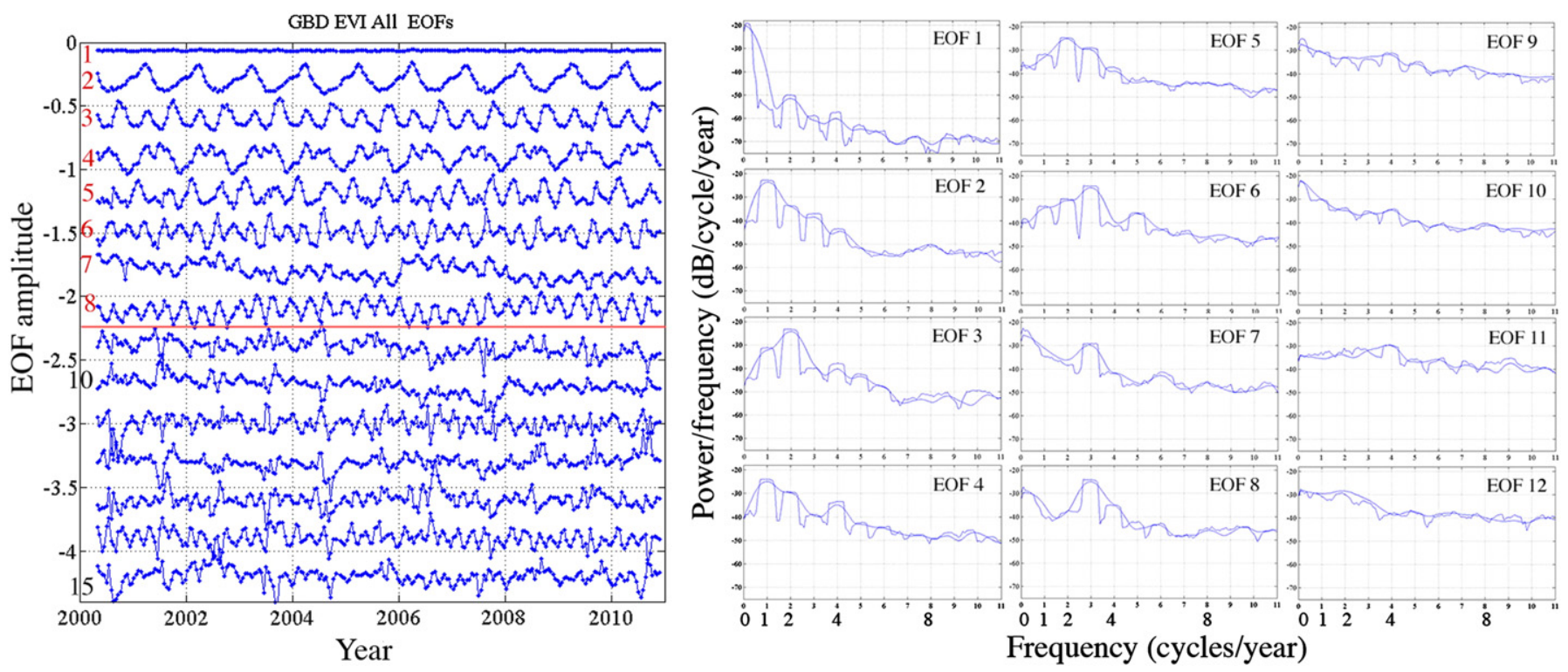

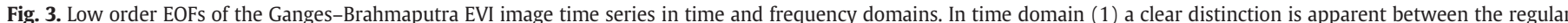

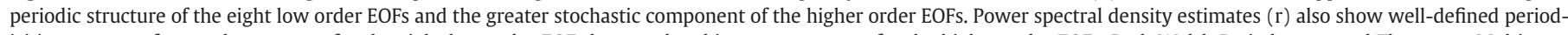

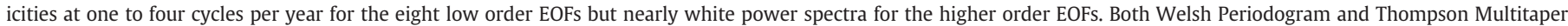
spectra estimates are in close agreement and identify the same peak frequencies.

aggregated regional statistic that effectively mixes the spatiotemporal patterns analogous to the way the spatial abundance of different temporal patterns determines which of these temporal patterns end up in the low order EOFs.

\subsection{Temporal mixture modeling}

Essentially, a model represents some aspect of reality. In the context of this study, modeling involves projection of the high dimensional data space onto a lower dimensional simplification represented by the model. In the approach described here, the mixture model represents the spatial and temporal patterns in the low order dimensions of the data. The relationships among the spatial PCs shows the relationships among the corresponding temporal EOFs. The topology of the pixel cloud in the temporal feature space comprised of the low order PCs shows how the individual EOFs are combined to represent the different temporal patterns in the data. The reason individual EOFs often convey little physical meaning when interpreted in isolation is because an individual pixel time series generally requires a combination of several EOFs to represent its structure. The combination of EOF weights and polarities associated with each pixel time series is given by its corresponding PC values. The variety of spatiotemporal patterns present in an image time series is therefore given by the combinations of EOFs (their weights and polarities) represented by the pixel cloud in the temporal feature space of the low order PCs.

The topology of the pixel cloud in the GBD EVI temporal feature space shows which combinations of EOFs exist in the data (and which do not) and the relative spatial abundance of the temporal patterns associated with different combinations of EOFs. By analogy to spectral mixing in spectral feature spaces, the temporal feature space conveys the spatiotemporal dimensionality of the data in terms of the distinct temporal endmembers and the continuum of temporal patterns that can be represented by various combinations of these endmembers. In this sense the topology of the cloud can resolve the degeneracy problem that complicates the interpretation of the EOFs. The topology of the pixel cloud in the temporal feature space reveals which specific combinations of low order EOFs are most distinct. The most distinct temporal patterns are the temporal endmembers associated with the apexes of the pixel cloud - in the same way the spectral endmembers occupy the apexes of the pixel cloud in spectral feature space. However, while the most "important" ST processes often control the structure of the covariance matrix (and hence its eigenstructure), not all processes of interest are necessarily contained in the low order dimensions. Locally important processes can end up relegated to the higher dimensions if they do not contribute sufficient variance to influence the covariance structure. Hence, the dimensionality of the feature space, represented in the eigenstructure of the covariance matrix, may be more accurately thought of as a continuum of diminishing influence rather than a discrete number.

The topology of the temporal feature space also illustrates the difficulty of interpreting individual EOFs. The eigenvalues indicate that the three low order dimensions corresponding to the temporal feature space in Fig. 5 represent 81\% of the spatiotemporal variance in the data. Yet all three low order PCs span the origin with both positive and negative values and almost all pixels have non-zero values for almost all PCs. This means that large numbers of pixel time series in the data are represented by combinations of EOFs of normal and reversed polarity. Although EOFs clearly do not "act alone" they are often interpreted individually in standard PC analyses. Ironically, it is both easier and more logical to interpret the temporal patterns in the data as combinations of EOFs rather than individual EOFs. The temporal feature space presents the temporal endmember (tEM) candidates as combinations of EOFs - eliminating the need to interpret individual EOFs.

The temporal feature space for the Ganges-Brahmaputra Delta has at least six distinct apexes in the three low order dimensions (Fig. 5). The apexes at either extreme of PC 1 correspond to areas with no vegetation (e.g. rivers) and areas with maximum EVI at all times (dummy values in the Bay of Bengal). These areas have no vegetation and therefore little or no temporal variability in EVI. The apexes at extremes of PC 2 correspond to areas with strong annual periodicities, but opposite phase, associated with EOF 2 and its mirror image. The third dimension of the temporal feature space reveals the biannual periodic structure associated with EOF 3 - also encompassing two opposing phases and all phase shifts in between as linear combinations. The apexes bounding the cloud in the PC2-PC3 projection therefore represent the four periodic temporal endmembers (tEMs) 

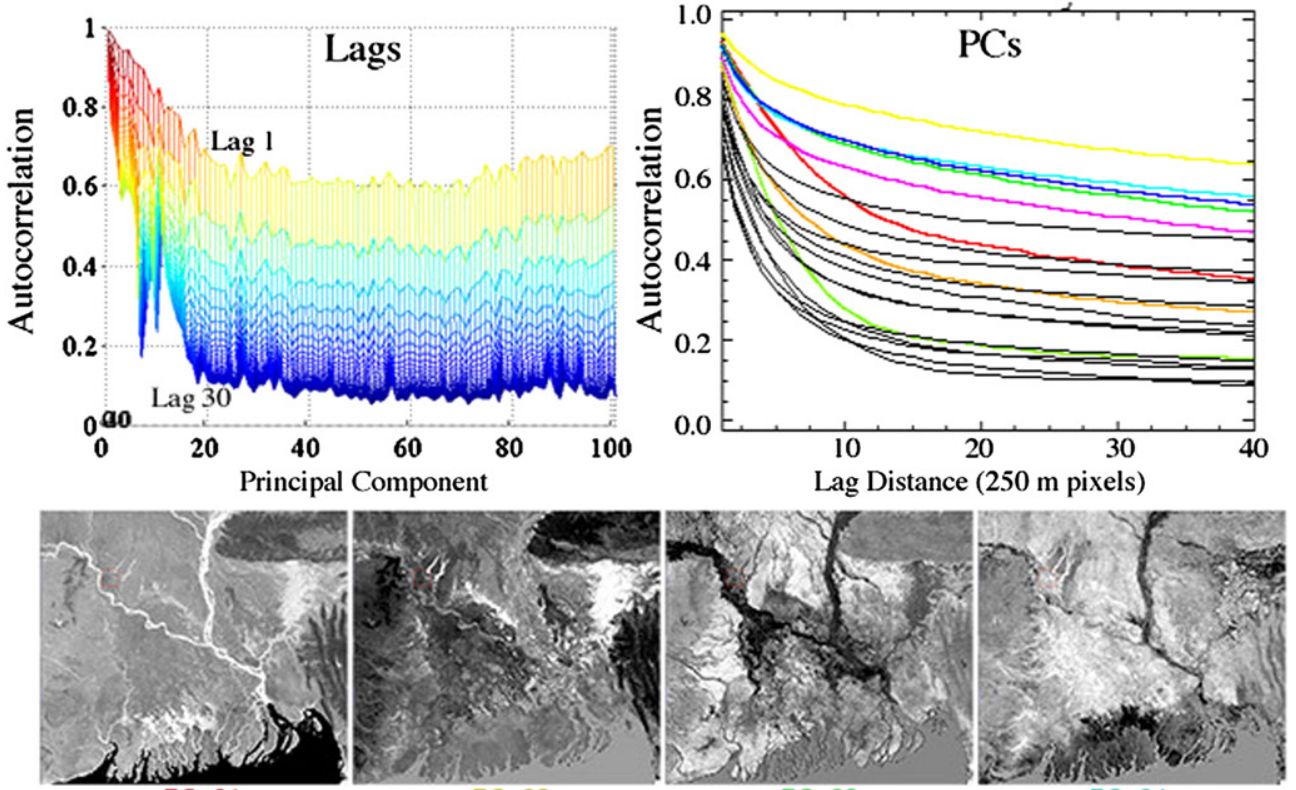

PC_01

PC_02

PC_03

PC 04
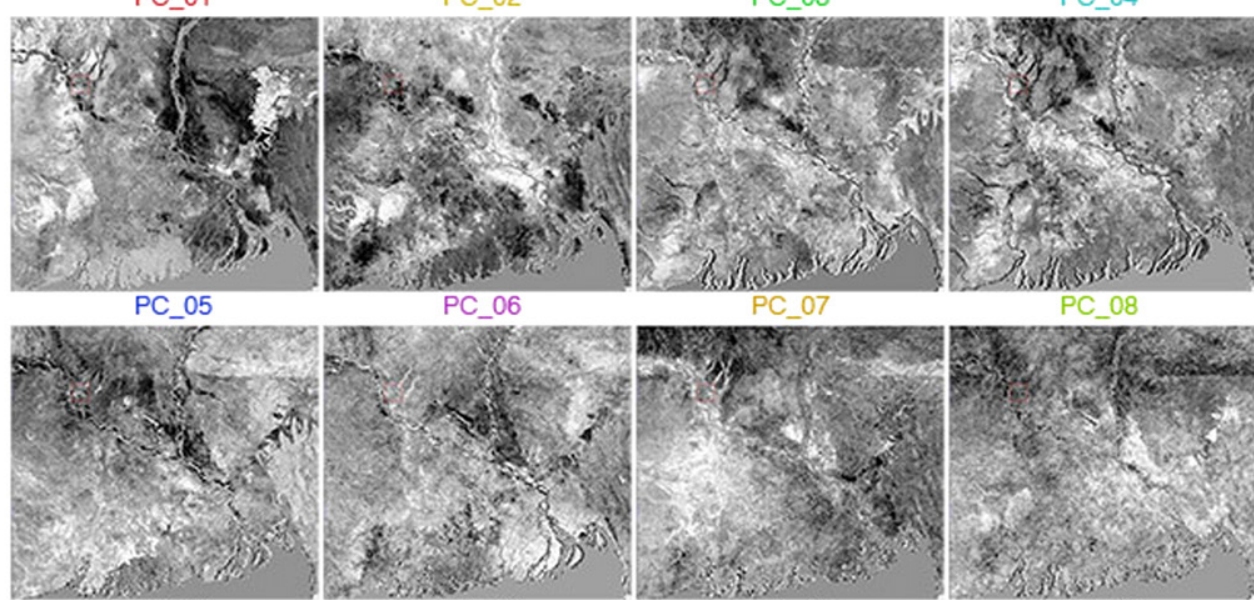

PC_06

PC_07

PC_08
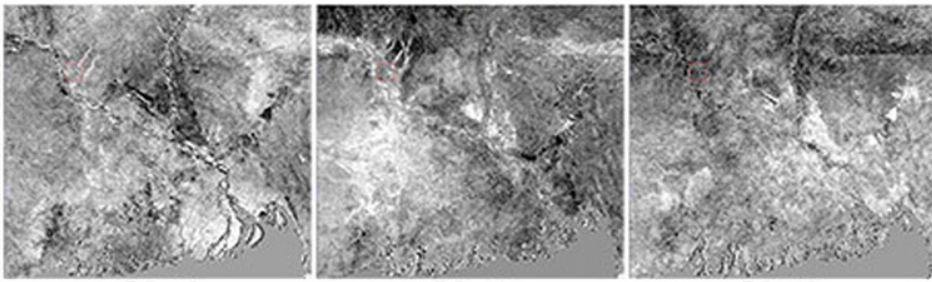

PC_09

PC_10

PC_11

PC_12
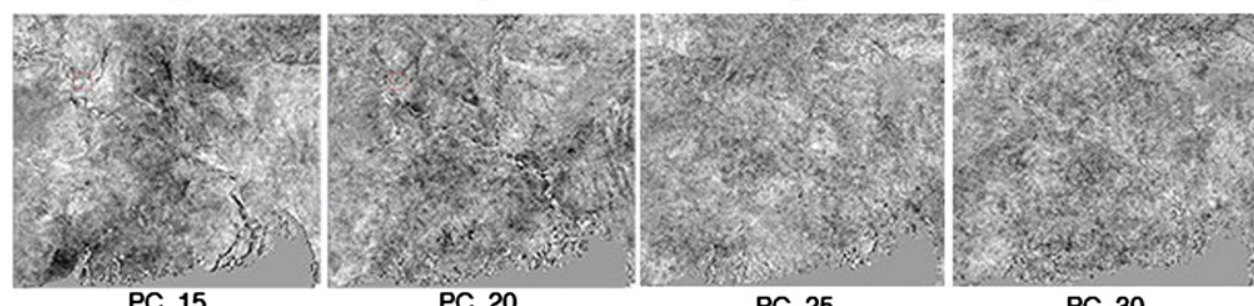

PC 30

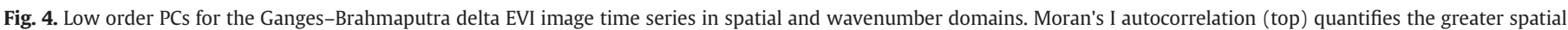

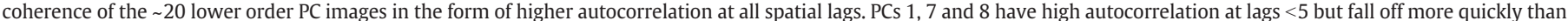
other PCs. For all lag distances, the autocorrelation falls off over the 20 low order PCs then stabilizes. ACF of other PCS have similar shape but diminish with dimension.

that describe most of the periodic variance in the data. The pixels within the convex hull circumscribing these four tEMs can be described as linear combinations of these tEMs in the same way that spectral mixtures can be represented as combinations of spectral EMs.

The selection of tEMs carries implicit assumptions about the processes being modeled. Larger numbers of tEMs can accommodate greater diversity of processes but can also result in overfitting if the tEMs chosen are not sufficiently independent. Larger numbers of tEMs can also make the results more difficult to interpret. In this analysis the tEMs are selected manually from the scatterplot - although statistical approaches developed for spectral mixture analysis could also be used(e.g. Monte Carlo Unmixing (Asner \& Lobell, 2000; Pixel Purity Index; Boardman, 1994)). Whatever method is used, it is important to use endmembers that are representative of the continuum of temporal patterns and not merely the samples furthest from the centroid of the pixel cloud. Anomalous and severely noise-contaminated time series generally reside at the periphery of the cloud. This highlights an important benefit to characterization and manual selection of tEMs. While automated methods can provide a statistically robust basis for EM selection, they can also be sensitive to outliers. Characterization and manual selection allow the analyst to determine the stability and physical plausibility of the EMs chosen - and implement the design of a specific model. 

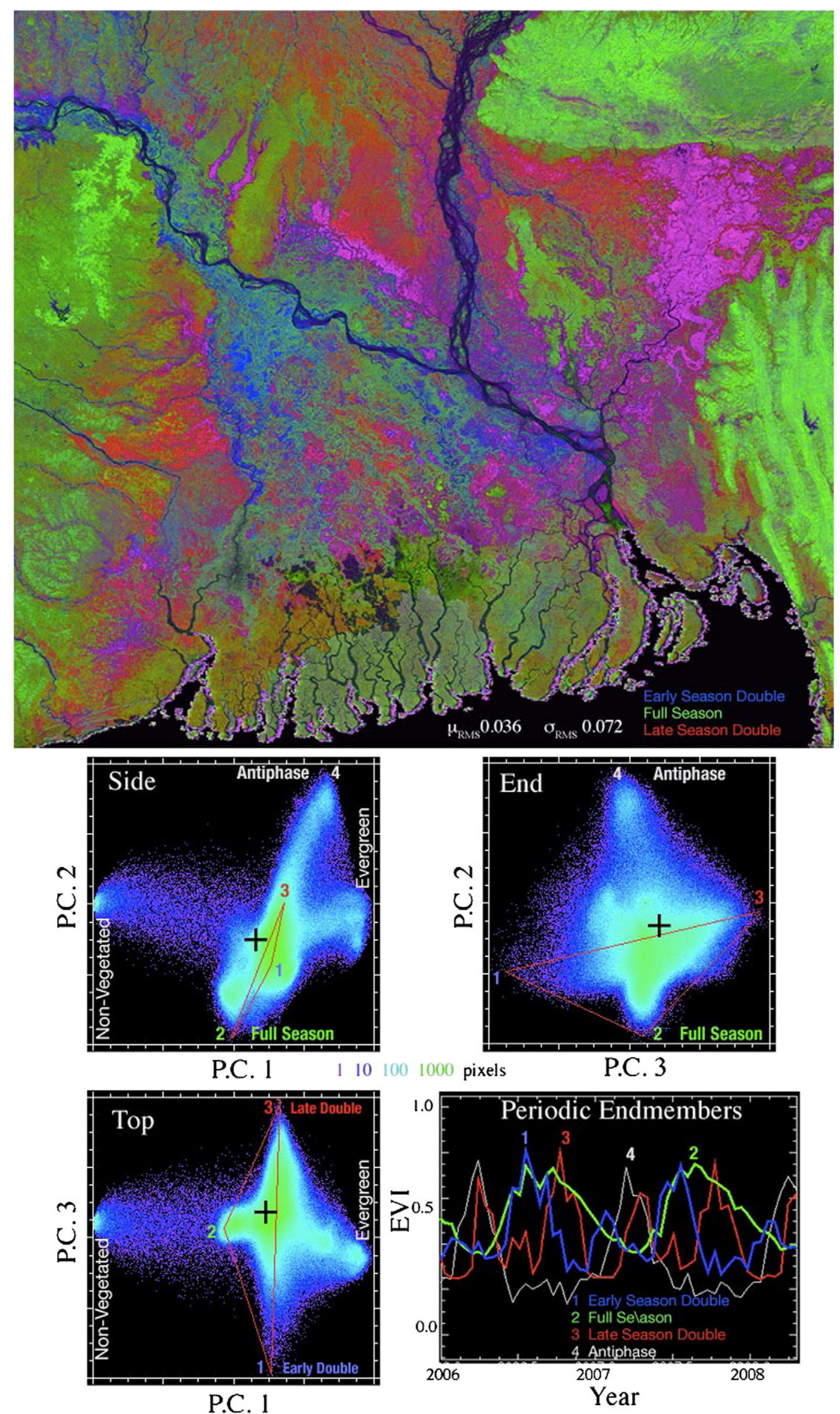


\subsection{Spatiotemporal phenology}

The variety of different vegetation phenologies on the GB delta can be represented as combinations of these periodic temporal endmember phenologies. The eigenstructure of the GBD EVI time series represents the amplitude of the phenological cycle with EOF 1 (multiplied by PC 1) and the different phases and periodicities as linear combinations of EOFs 2 and 3. Hence, the pixels with annual and subannual phenologies are clustered near the PC 2-3 plane (Fig. 5). The distinction between the annual and subannual phenologies is apparent in the crosslike structure of the cloud in the PC 2-3 projection.

To carry the analogy with spectral mixture analysis to its logical conclusion, the tEMs can be used in a linear mixture model to represent all time series in the data as linear combinations of the tEMs. As pointed out by (Lobell \& Asner, 2004), in the case of nonlinear difference ratios like EVI, the mixing between EMs is not actually linear in area. In this case, the temporal mixture fractions are merely measures of similarity to different tEMs - not necessarily areal fractions. Because inversion of the mixing equations is analogous to reprojection of the pixel cloud into the convex hull spanned by the tEMs (Boardman, 1990), the tEM fractions are actually measures of relative Euclidian proximity (or similarity) of any pixel time series in the cloud to each of the tEMs. For applications where areal fractions are required this type of characterization could be applied to individual spectral bands (rather than EVI) to select tEMs as part of a proper fraction estimation like that proposed by Lobell and Asner (2004). The use of spectral mixture fractions rather than nonlinear vegetation indices can also resolve this problem since mixture fractions scale linearly in area (Small \& Lu, 2006).

In simplest terms, the phenology of the GB delta can be represented in terms of annual and biannual cycles. The eigenstructure and topology of the temporal feature space indicates that most of the periodic variance is represented in the 2 nd and 3rd dimensions spanned by EOFs 2 and 3 . The pixels clustered near this plane form four apexes associated with temporal endmembers having two annual and two biannual periodicities. Candidate tEMs residing at these apexes are shown in Fig. 5. However, inversion of a four endmember linear model using these endmembers yields non-physical results in the form of negative fractions for each of the four endmembers. This is a manifestation of overfitting as the inversion literally inverts (flips) endmembers to obtain a smaller misfit by constructive and destructive interference of tEMs. In this case, the overfitting results from the similarity of the annual cycles in the deciduous vegetation in the highlands and the anti-phase wetland vegetation in the Syllhet basin. This overfitting can be reduced or eliminated by choosing either more or fewer or different tEMs. For simplicity, the effect of using fewer tEMs is illustrated here. Because the antiphase annual endmember represents a less important phenological endmember associated primarily with the Syllhet basin, it can be removed from the model. The resulting three endmember model is well-posed and returns positive fractions for all areas - except those associated with the antiphase endmember (magenta in Fig. 5). These pixels still have negative fractions for the annual endmember but the resulting fractions for the other pixels are positive. Removing the antiphase annual endmember stabilizes the inversion at the expense of the least relevant tEM. However, pixels with significant fractions of the antiphase tEM are still easily identified by their higher RMS misfit to the model. The choice of tEMs and design of the model depends on the application. For the GB delta phenology the tradeoffs between different models are discussed in detail in a separate study incorporating field observations (Small et al., In Prep.).

The point of this example is to illustrate 1 ) a relatively clear distinction between periodic and aperiodic dimensionality, 2) the utility of the temporal feature space for identification of tEMs and 3) the ability of a very simple three tEM model to represent the salient spatiotemporal features of a relatively complex spatiotemporal environment. The utility of the three tEM model is to illustrate how a combination of processes can be identified and represented spatially and temporally with a relatively simple model. The development and validation of multiple, application-specific, models for the GB delta is described in detail in a separate study (Small et al., In Prep.). The objective here is not to identify specific crops but to characterize the number of distinct aggregate phenologies and their influence on the temporal feature space. Using inter-annual endmembers captures the regional inter-annual variability on the basis of regional spatial abundance. A comparison of results obtained from multiple annual analyses and the single inter-annual analysis are presented in the more detailed region-specific study.

\subsection{Projection filtering}

In cases where the eigenstructure allows for separation of deterministic and stochastic variance the eigenstructure also suggests a solution to the problem of endmember variability. The clear distinction between the low order periodic structure and the stochastic variability superimposed on it suggests that much of the aperiodic noise can be removed by inverse transforming only the 8 low order dimensions of the PC transformed data. By inverting the PC rotation for only the periodic modes the back projection effectively filters out the aperiodic component of the variance. Because the inverse rotation of the subspace removes aperiodic "noise" by projecting the selected dimensions back onto the original time axes it is referred to here as projection filtering. This is similar to the approach using the Minimum Noise Fraction proposed by (Boardman \& Kruse, 1994) The effect on the three periodic tEMs of Fig. 5 is shown in Fig. 6. The projection filtered tEMs are noticeably smoother but the smoothing is achieved by retaining the periodic structure derived from the entire data set. In a sense, this achieves a similar averaging to the Monte Carlo method of tEM selection proposed by Lobell and Asner (2004) but uses the eigenstructure of the covariance as a basis for the averaging. In addition to reducing spurious tEM variability, projection filtering can aid in interpretation of spatiotemporal patterns. By eliminating the aperiodic variance from each time series, projection filtering increases the spatiotemporal continuity of the dominant periodicities. The benefit of projection filtering to interpretation of spatiotemporal processes is illustrated by comparison of the Time-Space cubes in Fig. 7.

\subsection{Multiscale structure: annual periodicities and inter-annual trends}

In addition to highlighting the dominant regional phenologies, the characterization also identifies some localized inter-annual patterns. The linear model described above uses only three low order PCs to represent the structure of the image time series but the tEMs selected from the temporal feature space can contain all of the dimensions - or a periodic subset. By using a small subset, the linear model can represent the spatiotemporal structure of the data in terms of the statistically dominant patterns in the low order dimensions. However, the eigenstructure reveals additional dimensions of uncorrelated variance. Of particular interest are the inter-annual components of dimensions 7 and 8 . The corresponding

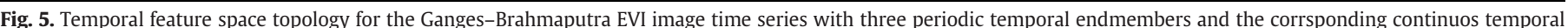

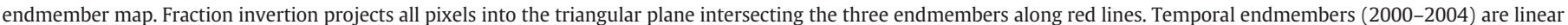

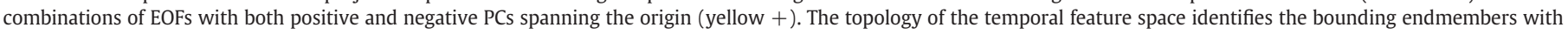
physically meaningful periodicities. 


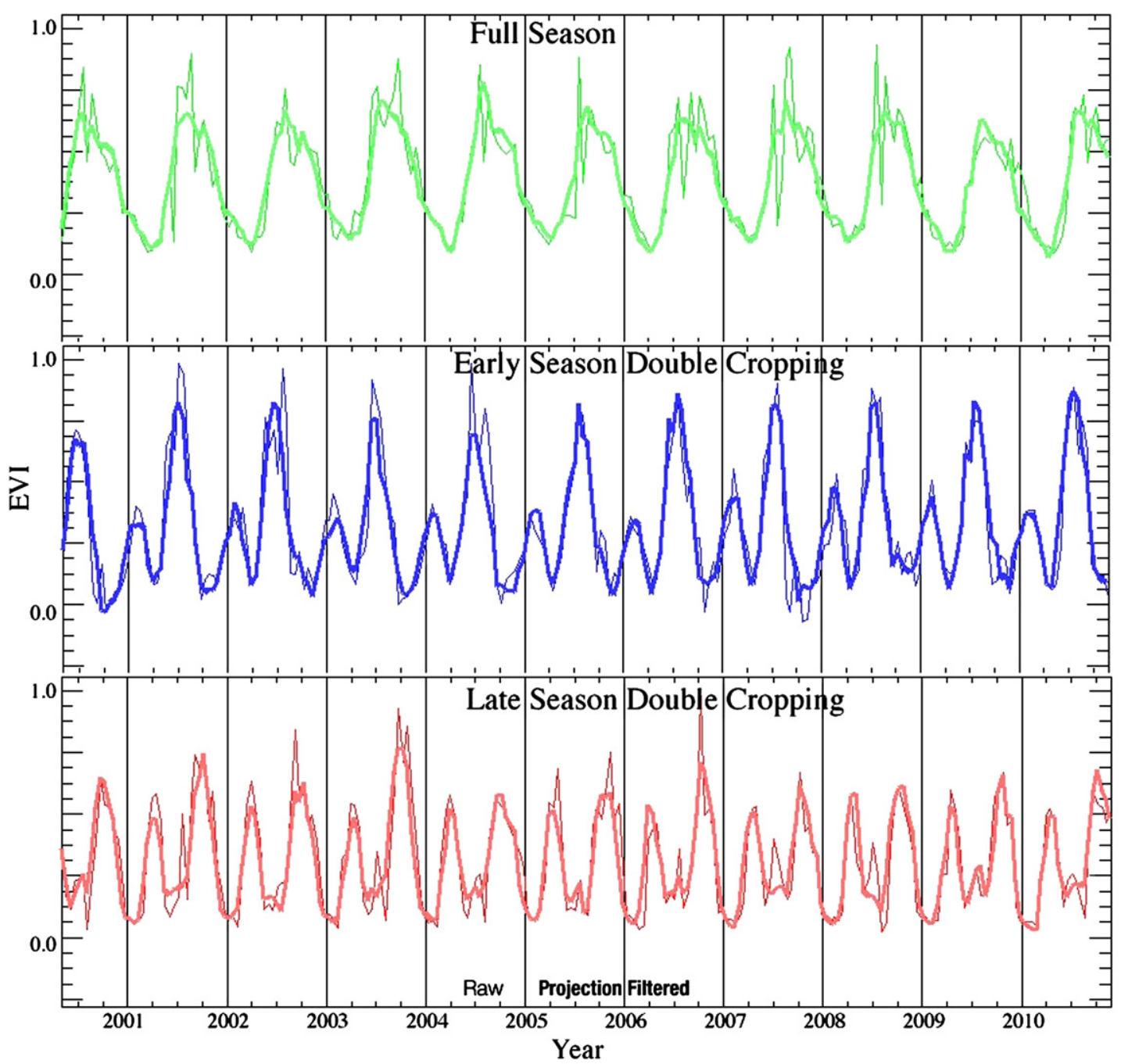

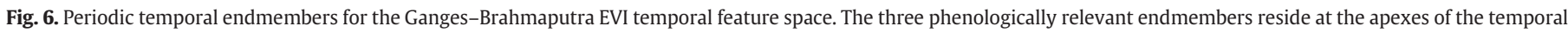

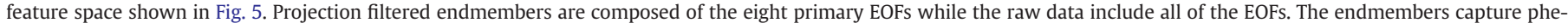
nological variations in both amplitude and phase as well as some degree of interannual variability.

2D subspace of the 8D temporal feature space shows a surprising structure (Fig. 8). Although the PCs are uncorrelated overall, the feature space of PCs 7 and 8 is clearly composed of correlated and uncorrelated limbs and an uncorrelated core. For pixels on the correlated limbs, the inter-annual trends in EOFs 7 and 8 cancel each other out. However, on the anticorrelated limbs the trends of the EOFs combine in phase to reinforce each other. In this way, the PC 7-8 subspace identifies pixels with strong trends of vegetation increase and decrease on inter-annual time scales. This pattern could result from progressive erosion and deposition over the course of several flood cycles. The geographic locations of these pixels correspond to areas on the banks of the migrating river channels (Fig. 8). During the annual cycle of rising and falling water levels, areas of sediment deposition would emerge sooner each year and remain exposed longer thereby allowing vegetation to become more established and resistant to erosion during subsequent floods. Conversely, areas of sediment erosion would remain under water longer each year thereby making it more difficult for seasonal vegetation to establish root systems sufficient to remain in place from year to year. In this example, the characterization revealed an unexpected feature in the data but the temporal mixture model would not provide the best tool for modeling it. The trend and change decomposition of (Verbesselt et al., 2010) would be better suited to modeling this type of feature.

\section{More challenging problems}

The example of phenology mapping with MODIS-derived vegetation indices is a relatively well-posed problem. In the GBD there are several very distinct phenologies and all are temporally oversampled by the 16 day composites. Despite the tropical cloud cover and monsoon, the periodic phenological signal is relatively large compared to the higher order aperiodic variance. However, there are many cases where the problem of spatiotemporal analysis of multitemporal imagery is not so well-posed. Two such examples are presented briefly to illustrate some of the challenges that can arise with this type of analysis. Both examples are discussed in greater detail in the references provided.

\subsection{Phenology mapping with Landsat: effects of temporal aliasing}

How accurately a spatiotemporal dataset represents the measured process(es) depends on both spatial and temporal sampling. Oversampling is a necessary, but not generally sufficient, characteristic of a well-posed estimation problem. Undersampling in either time or space can result in aliasing in which the spatial and temporal structure of the data misrepresent the processes they measure. This can lead to an ill-posed estimation problem where at least some of the spatial and temporal characteristics of the process cannot be 


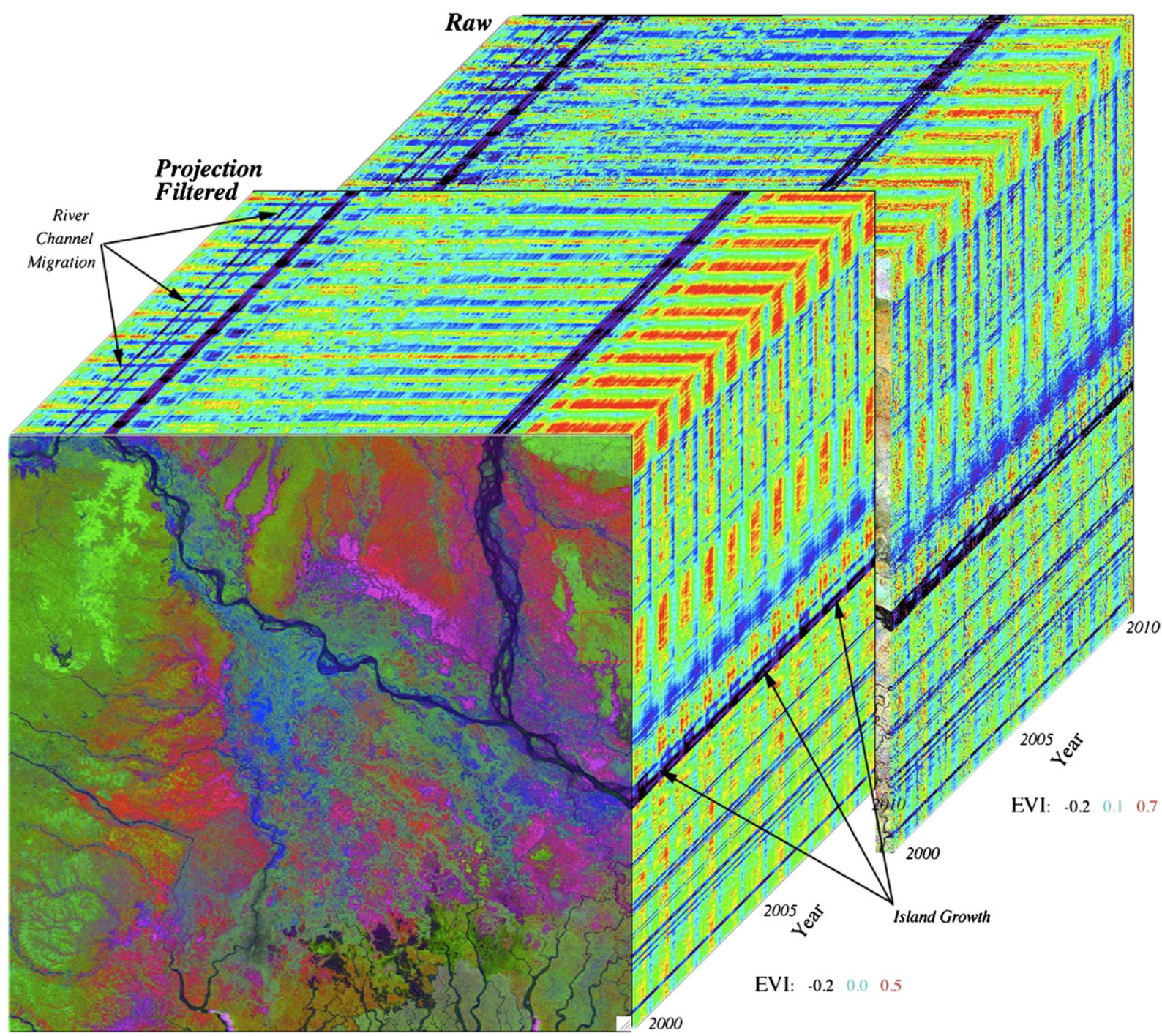

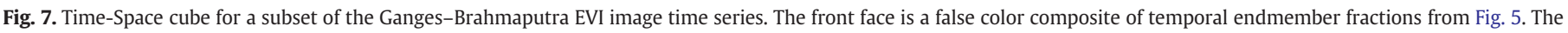

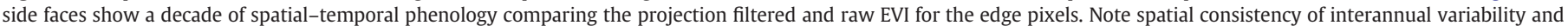
interannual evolution of areas near the rivers.

accurately derived from the data. The previous example of seasonal phenology mapping with 16 day composites illustrates a well-posed problem because the dominant periodicities are all oversampled in time. In contrast, phenology estimation from single year Landsat data is vulnerable to serious temporal aliasing because the combination of cloud cover and biweekly revisit times often does not allow for accurate representation of green-up and senescence phases. The use of Landsat for phenology mapping has been explored thoroughly by (Fisher \& Mustard, 2007; Fisher et al., 2006). The impressive results of these studies demonstrate the power of Landsat for phenology mapping - but the approach is based on prescriptive modeling and thus fundamentally different from the characterization discussed in this study. In many areas, inter-annual variability and limited temporal coverage could present a greater problem than it did in the analyses of Fisher and Mustard (2007). The following example illustrates how Time-Space characterization can be used to distinguish between seasonal and inter-annual changes in vegetation abundance - even in the presence of temporal aliasing.

In many parts of the world the Landsat coverage of any one year is not sufficient to characterize the phenology cycle, but the combination of several years can represent the annual green-up and senescence - although contaminated by inter-annual variability and phase shifts in the transitions. In the northeastern US the primary sources of inter-annual variability are changes in the onset of spring green up, occurrence of summer drought and duration of autumn senescence. For this example, we use 97 cloud-free images of New York City collected on different julian days over 25 years. Vegetation fractions are estimated using generic spectral endmembers (Small, 2004) with a unit sum constrained linear mixture model applied to scenes of calibrated exoatmospheric reflectance. Seasonal variations in structural shadow have not been removed but are largely contained in the dark fraction component. The spatiotemporal dynamics are illustrated here using only the vegetation fractions arranged by julian day to form a composite annual time series.

The eigenstructure of the NYC vegetation fraction stack shows the combined effect of seasonal and inter-annual vegetation dynamics. The correlation matrix structure clearly shows the distinction between deciduous leaf-on and leaf-off periods - and gives some indication of the inter-annual variability in green up and senescence phases (Fig. 9, inset). In contrast to the previous example, the eigenvectors of these data show less structure - suggesting lower dimensionality. The eigenvalue spectrum shows at most 5 dimensions. When the fractions are arranged by Julian day EOFs 1 and 2 show clear annual phenology modes - although inverted - while higher order EOFs are more difficult to interpret. However, when the image stack (and resulting EOFs) is sorted by year EOFs 3 \& 4 show decadal 
a

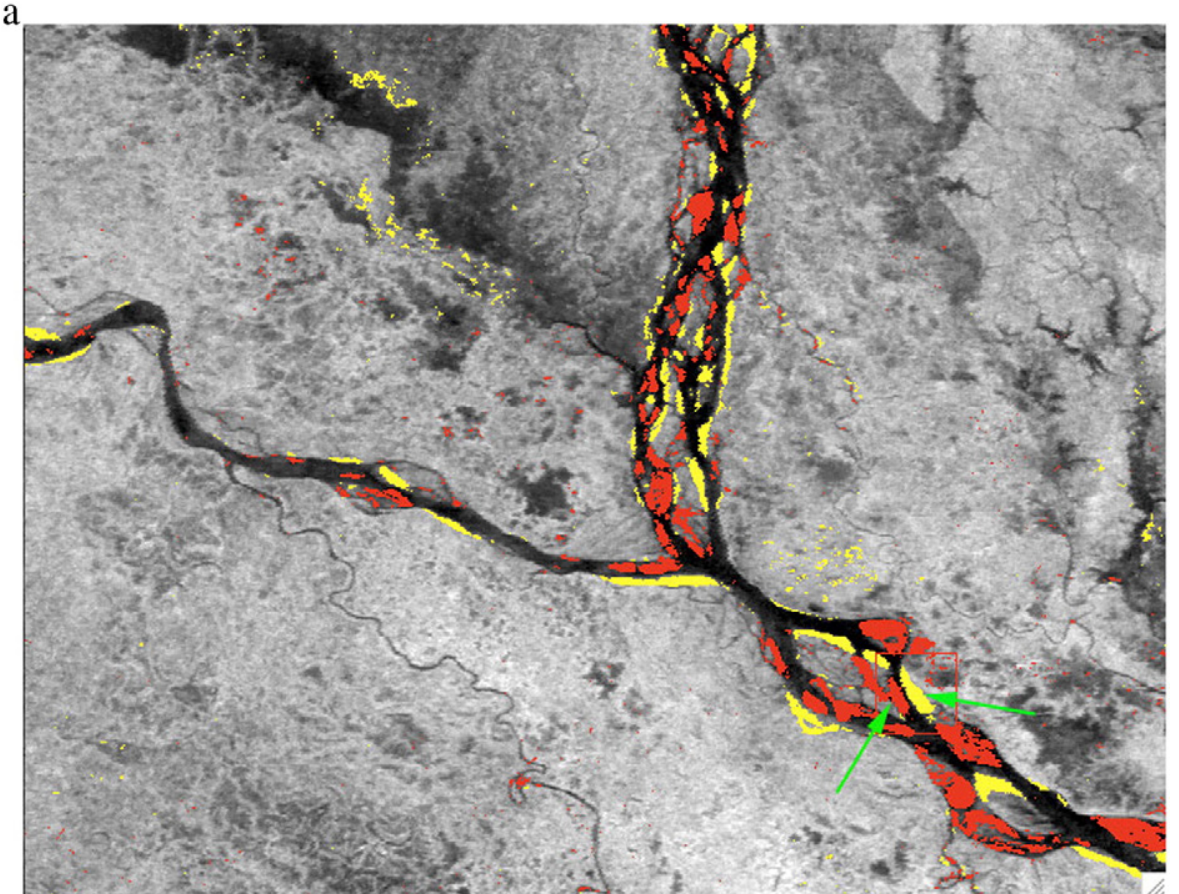

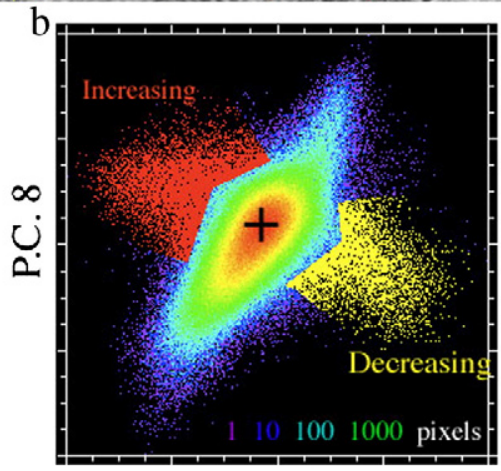

P.C. 7

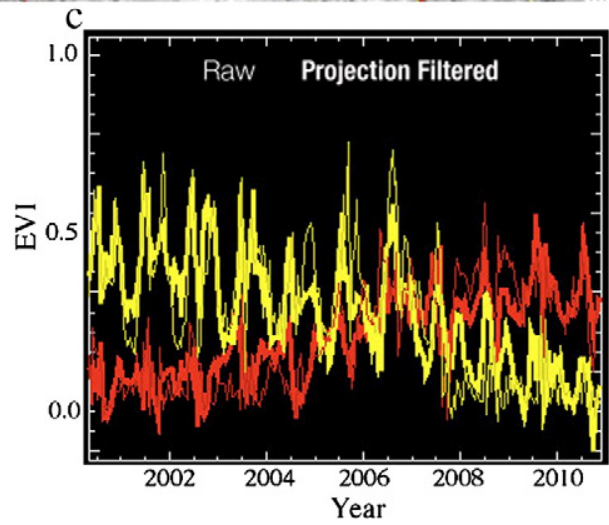

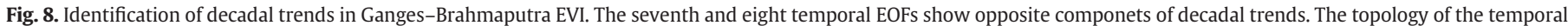

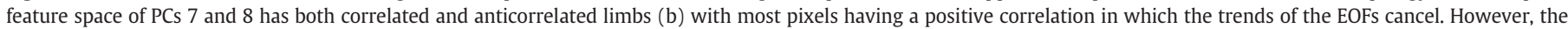

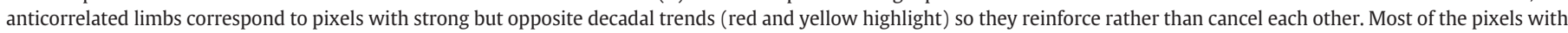

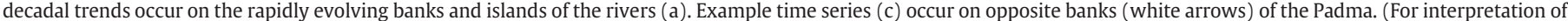
the references to color in this figure legend, the reader is referred to the web version of this article.)

trends (Fig. 9). In the spatial domain the PCs offer little help as the structure of all PCs $>2$ are either incoherent or dominated by structure localized in wetlands.

The temporal feature space lends insight into the spatiotemporal structure of the data. The projection of PCs 1 and 2 shows a well-defined triangular structure with sharp apexes (Fig. 9) - topologically similar to that usually seen in spectrally diverse imagery containing rock and soil substrate, vegetation and dark or shaded surface. The three most prominent apexes are distinct with 2 apexes at negative PC1 having clear phenological patterns (grass \& wetlands) and one at the positive PC 1 end showing no annual structure. A 3rd apex is apparent near an isolated cluster between the two negative PC 1 apexes. Although intermediate between the other two vegetated apexes, it occurs on a strong directional mixing trend with the unvegetated apex.

In the temporal feature space the spatiotemporal structure and phenology are clear. Comparison of annual vegetation fraction profiles of the pixels at the apexes of the temporal feature space shows a pronounced difference between the persistently green grass and evergreen endmember (red) and the other two seasonal tEMs with a bimodal oscillation between dense and absent vegetation (Fig. 9). There is a similarity between the early green up deciduous (green) the later green up wetland vegetation (blue) tEMs but with a distinct phase shift and longer period for the earlier greening deciduous (green) endmember. The PC $3 / 2$ projection in Fig. 9 shows a conspicuous protruberance. The corresponding PC 3 is spatially incoherent with no obvious interpretation in terms of phenology. This feature is related to inter-annual changes in areas where vegetation was cleared for development. In this example the Time-Space characterization shows the relatively low dimensionality of the data while the temporal feature space separates the seasonal and inter-annual components of change and provides physically meaningful tEMs. The phonological mixture model derived from this analysis is discussed in greater detail by Small and Lu (In Prep. 2012).

\subsection{Mapping decadal change in night light: limitations of mixture models}

The third example uses a time series of annual composites of night light brightness to illustrate the utility of Time-Space characterization in a case where a temporal mixture model in not necessarily appropriate for representing the data. The Defense Meteorological Satellite Program (DMSP) Operational Linescan System (OLS) sensors have 

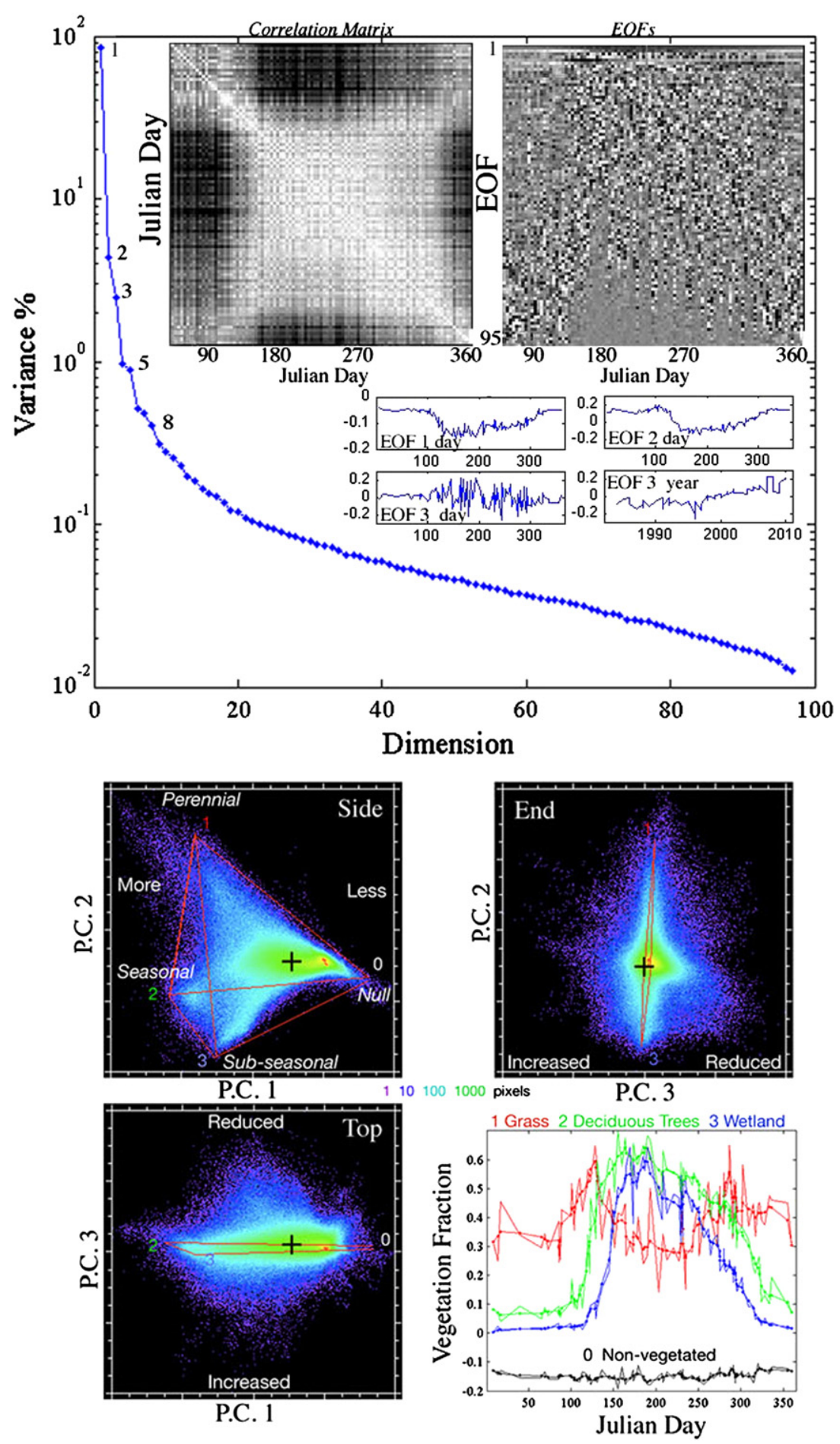

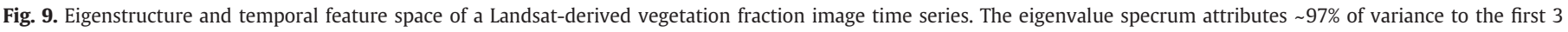

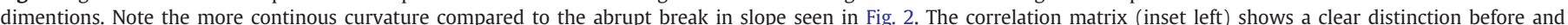

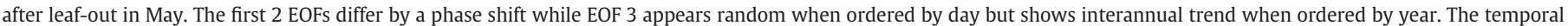
feature space shows clear apexes with tEMs corresponding to distinct phennologies and interannual change trends off plane in dimension 3.

imaged emitted night light from Earth's surface since the 1970's. Temporal overlap in the missions of 5 OLS sensors allows for intercalibration of 30 annual composites over the past 19 years (Elvidge et al., 2009). The resulting image time series captures a spatiotemporal signature of the growth and evolution of lighted human settlements and development. The most pronounced changes during this time have occurred in Asia. Urban growth and development in Asia have resulted in increasing number and spatial extent of lighted development (Small \& Elvidge, in press). However, the geographic diversity of the region, combined with multiple cycles of economic growth, have produced a wide variety of temporal trajectories in night light brightness and extent. Before modeling these trajectories, it is important to characterize the diversity of different spatial and temporal patterns present in the data.

The eigenstructure of the Asia night light image time series suggests a relatively low spatiotemporal dimensionality. The eigenvalue spectrum attributes $91 \%$ of the variance to the first two dimensions with incrementally diminishing variance for the remaining 28 


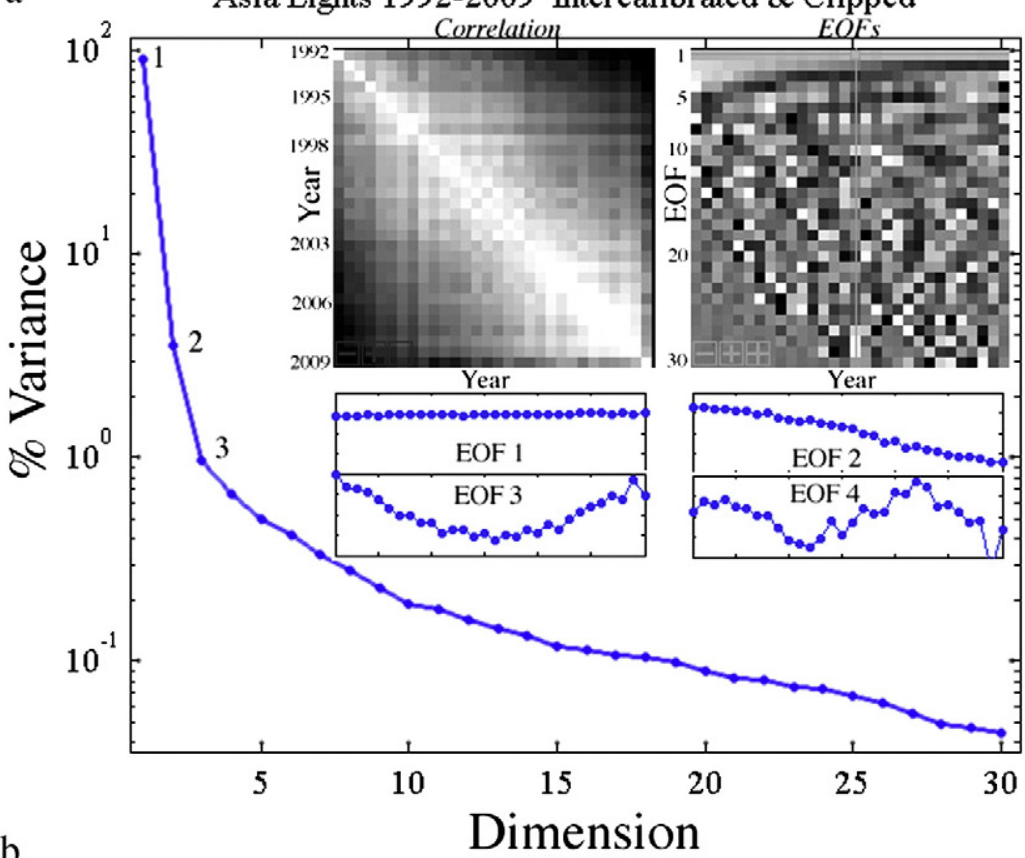

b
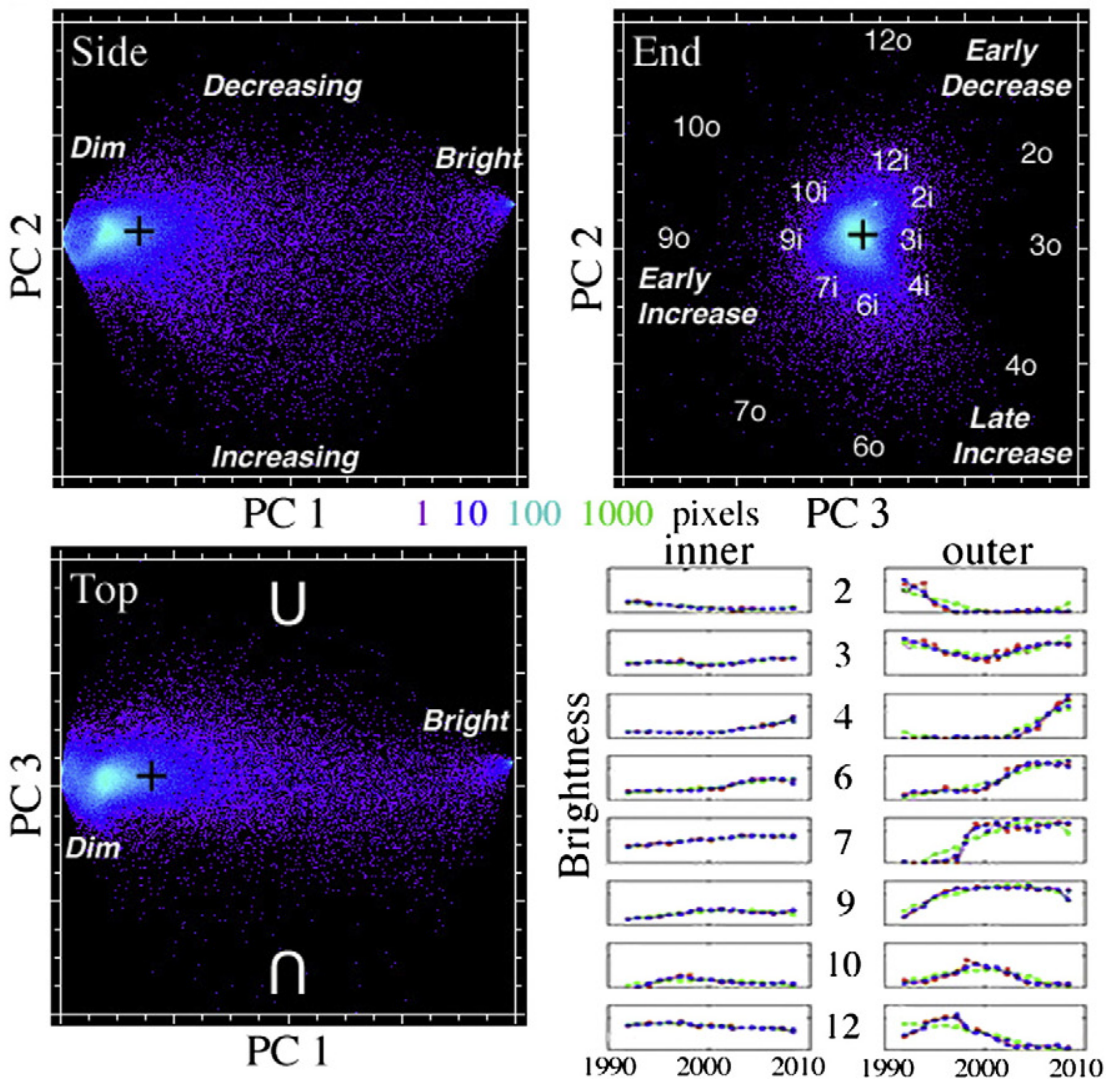

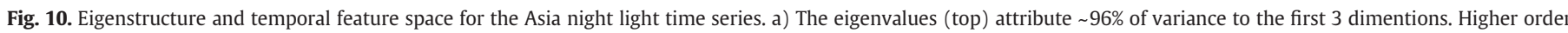

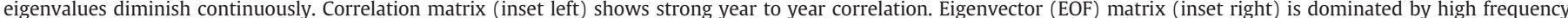

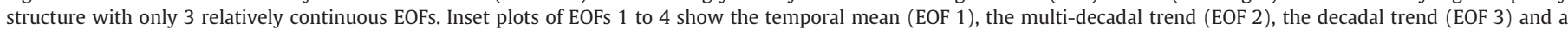

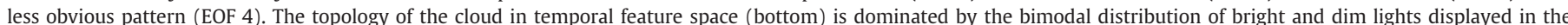

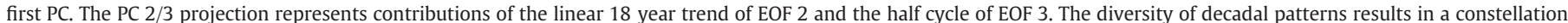
of distinct EOF 2/3 combinations surrounding the bimodal brightness axis in PC1. Inset numbers on End View correspond to time series in Fig. 3.

dimensions (Fig. 10). The correlation matrix is dominated by high year to year correlations - with the exception of the F15 2008 composite (subsequently found to be corrupted and removed from the current version of the dataset). The eigenvector matrix shows a rapid transition to incoherent high frequency structure beyond the first 3 or 4 EOFs (Fig. 10).

Comparison of the spatial PCs and temporal EOFs illustrates the spatiotemporal dimensionality of the image time series with a 
very clear distinction between the first three dimensions and the higher order dimensions (Fig. 10). The problem of degeneracy is manifest in the opposing linear trends of the first two EOFs. The first EOF is nearly constant in time (although with a slight positive trend) while the corresponding spatial PC shows the temporal mean brightness. The second EOF takes the form of a slightly sigmoid diminishing decadal trend. The third EOF takes the form of reversing decadal trends - an unusual pattern for night light brightness in either positive or negative polarity. The higher order dimensions become increasingly difficult to interpret in either the temporal EOFs or spatial PCs as both are increasingly dominated by high frequency variance.

As in previous examples, the topology of the pixel cloud in the temporal feature space provides additional insight into spatiotemporal structure of the dataset. The PC1-PC2 projection illustrates bimodal distribution of bright and dim lights that dominates the variance structure of the data. The PC1-PC3 projection is very similar to the PC1-PC2 projection revealing a bimodal axisymmetric structure to the low order 3D feature space. In strong contrast to the previous examples, the PC2-PC3 projection lacks apexes and linear trends. The topology of the pixel cloud in this projection is nearly circular but with a slight indentation on the positive PC3 side.

The lack of apexes and linear trends in the PC3-PC2 projection of the temporal feature space illustrates the diversity of temporal change patterns present in the data. Despite the absence of linear mixing trends, the structure of the temporal feature space does convey information about the temporal patterns in the night light data. This is apparent when considering the temporal patterns that result from varying combinations of EOFs 2 and 3. The relationship between the structure of the feature space and the form of temporal trajectories is illustrated by the inset (lower right) in Fig. 10. Together EOFs 2 and 3 represent progressive changes in the sense and timing of decadal changes in night light. The varying combinations of EOFs 2 and 3 represent a continuum of progressive phase shifts in the same way that sinusoidal phase shifts can be represented as a continuum of $\mathrm{x} / \mathrm{y}$ ratios on the unit circle.

The effects of projection filtering on the profiles in Fig. 10 further illustrates the spatiotemporal dimensionality of the dataset. Raw trajectories (red) are generally well represented with 6 low order EOFs and frequently with only 3 . The smoother decadal variations are captured in the first three dimensions but in some cases more abrupt changes at shorter time scales require additional dimensions to capture their sharper structure. In this example the circular topology of the temporal feature space suggests the unsuitability of the linear mixture model - but also reveals the underlying structure of the temporal patterns of change. By separating the overall brightness in dimension 1 from the decadal trends in dimensions 2 and 3 the characterization provides a basis for identifying and mapping different temporal patterns of decadal change in night light without the need to either saturate the brightly lit areas or attenuate the more dimly lit areas (Small \& Elvidge, in press).

This example illustrates an important limitation of the EOF analysis. The processes driving development and changes in anthropogenic night light are not dominated by periodicities like those in the phenology examples. Because increases and decreases in night light can and do occur in any year of the time series, the diversity of temporal trajectories is large compared to the dimensionality of the data. In other words, the night light data are not dominated by a relatively small number of temporal patterns but rather contain a large number of patterns that can be represented with a small number (3) of dimensions. EOFs 2 and 3 combine to represent a wide variety of different patterns (Fig. 10 inset lower right) but they do not combine in a simple binary mixing trend. Similar structure is seen in MODIS -derived vegetation indices where a "phenological wave" propagates over a region producing a spatiotemporal phase shift a similar pattern appears at different places at different times. An example is the green up following the monsoon in south Asia or the green up following the northward movement of the InterTropical Convergence Zone in the African Sahel. While not necessarily linear, the interference of two periodic EOFs can be used to represent the spatiotemporal propagation of a similar pattern through a dataset analogous to the way phase and amplitude are represented in the frequency domain.

\section{Discussion and conclusions}

The combination of characterization and modeling described here is potentially very powerful for identifying and representing spatiotemporal processes - but is not well suited to many change detection problems. The EOF analysis can represent the covariance structure of a high dimensional dataset with a relatively small number of spatial and temporal patterns but these patterns are not always the most interesting or important features in the dataset. Discrete abrupt changes will not generally be captured in the low order dimensions unless they are large enough and pervasive enough to exert influence on the covariance structure. Similarly, the same short period temporal pattern occurring at different times can require a large number of dimensions to be represented accurately - or may be represented by progressive interference of two or more EOFs as described above. In either of these cases the linear mixture model is not necessarily an effective way to represent the feature. Also, like any PCA-based approach, the results are data dependent so the result for one geographic region is not necessarily applicable to another. In this sense, the specificity of the EOFs can suffer from a lack of generality or portability. Multiscale analyses encompassing the entire domain of the data (e.g. global MODIS coverage) or a suitably diverse collection of data can potentially show some convergence in the structure of the feature space (e.g. (Small, 2004)) but this scale of analysis is likely impractical for many spatiotemporal applications. Particularly given the potentially vast number of temporal patterns that may exist in some datasets. The point of the three contrasting examples is to illustrate the range of possible outcomes from this type of analysis. One important benefit of this method is the relative ease of implementation. The PC transformation is a relatively quick and inexpensive way to explore the structure and dimensionality of large datasets. However, it is important to be aware of the limitations of the tool from the outset.

The characterization described here differs from traditional PC and EOF analyses in its use of the temporal feature space and mixture model to resolve the problems of degeneracy and to avoid the need to interpret individual EOFs. It also differs from most traditional EOF analyses in the characterization of temporal rather than spatial modes of variability. In many geophysical systems temporal variance is correlated with spatial scale; larger patterns are associated with greater temporal variance than smaller features. This is particularly true when mass and energy considerations require some degree of spatial connectivity as with ocean or atmospheric circulation. In contrast, land cover can impose relatively fine scale structure on the landscape that can effectively decouple spatial and temporal scales of variance. Vegetation phenology can have a wide range of spatial and temporal scales. Regional climate, soil types and ecological processes acting on the distribution of indigenous vegetation may result in large areas being modulated by annual to inter-annual phenological patterns whereas human intervention in the form of irrigation and agriculture can superimpose higher temporal frequency variance of lesser or greater amplitude at spatial scales many orders of magnitude finer in spatial dimension. For this reason, it often makes more sense to characterize spatiotemporal variance structure in terms of temporal patterns before subsequent interpretation of the spatial structure of these patterns. 
The temporal mixture analysis described here differs from previous studies using temporal mixture models in its use of the eigenstructure to characterize the dimensionality and in its use of the temporal feature space to inform the design of the model and selection of endmembers. The most relevant comparison is with the temporal mixture modeling of phenology described by Lobell and Asner (2004). The approach described by Lobell and Asner (2004) uses temporal endmembers based on single year phenological patterns selected using ancillary information (Landsat) and does not include any characterization of the temporal patterns present in the MODIS data. The approach described here uses temporal endmembers based on multi-year phenological patterns selected using the characterization of the temporal feature space and the complexity of the desired model. The two approaches are fundamentally different but not mutually exclusive. The characterization described here could inform the modeling approach proposed by Lobell and Asner (2004). The approach given by Lobell and Asner (2004) may be more appropriate for identification of temporal patterns known apriori and identifiable using ancillary information (as with agriculture) whereas the approach described in this study may be more appropriate for characterization of phenology in areas where the temporal endmembers are not known apriori and a more general representation is needed.

This comparative analysis highlights some important differences and similarities between temporal mixture modeling and spectral mixture analysis. One fundamental difference is the nature of the processes being modeled. Spectral mixture models simulate the process of radiance field measurement by sensors. The physical processes involved can be described more explicitly with radiative transfer models and sensor models but in many cases simplified to a linear mixture model that accurately represents the aggregate net effect of these other processes. Temporal mixture models are not limited to radiative transfer and can be generalized to include many more types of processes than spatial aggregation of the radiance field in the IFOV. While the physical process of radiance field mixing remains relevant for vegetation phenology, the night light example illustrates a more general type of process where temporal patterns are not necessarily spatial mixtures of a few endmembers and one physical process. Despite their greater generality, and potential complexity, this larger class of problems can still benefit from being described by their fundamental spatial components and common temporal patterns. In much the same way that Fourier analysis can be generalized beyond the process of wave propagation to represent a more general class of periodic process, the use of EOFs derived from a variety of component transformations to represent spatiotemporal processes can be used to understand a wide range of spatiotemporal processes. By generalizing the procedures used for spectral mixture analysis, and combining them with the concept of deterministic and stochastic dimensionality from EOF analysis, it is possible to use characterization and modeling together to understand more complex high dimensional systems.

\section{Acknowledgments}

This work was supported by a grant from the National Science Foundation and the United States Forest Service (NSF-DEB0948451), the New York City Department of Environmental Protection and the Doherty Foundation. Copious thanks to Doug Martinson, Alexey Kaplan and Alessandra Giannini for thought-provoking discussions and three anonymous reviewers for helpful suggestions.

\section{References}

Adams, J. B., Smith, M. O., \& Johnson, P. E. (1986). Spectral mixture modeling; a new analysis of rock and soil types at the Viking Lander 1 site. Journal of Geophysical Research, 91, 8098-8122.
Asner, G. P., \& Lobell, D. B. (2000). A biogeophysical approach for automated SWIR unmixing of soils and vegetation. Remote Sensing of Environment, 74, 99-112.

Bellman, R. E. (1957). Dynamic programming. : Princeton University Press.

Boardman, J. W. (1989). Inversion of imaging spectrometry data using singular value decomposition. IGARSS'89 12th Canadian Symposium on Remote Sensing (pp. 2069-2072). Vancouver, B.C.: IEEE.

Boardman, J. (1990). Inversion of high spectral resolution data. SPIE - Imaging Spec troscopy of the Terrestrial Environment, 1298, 222-233.

Boardman, J. W. (1994). Geometric mixture analysis of imaging spectrometery data. Proc. Int. Geoscience and Remote Sensing Symp., 4. (pp. 2369-2371).

Boardman, J. W., \& Kruse, F. A. (1994). Automated spectral analysis: a geologic example using AVIRIS data, north Grapevine mountains, Nevada. Tenth Thematic Conference on Geologic Remote Sensing (pp. I407-I418). Ann Arbor, MI: Environmental Research Institute of Michigan.

Bretherton, C. S., Smith, C., \& Wallace, J. M. (1992). An intercomparison of methods for finding coupled patterns in climate data. Journal of Climate, 5, 541-560.

Byrne, G. F., Crapper, P. F., \& Mayo, K. K. (1980). Monitoring land-cover change by principal component analysis of mulittemporal Landsat data. Remote Sensing of Environment, 10, 175-184.

Coppin, P. R., \& Bauer, M. E. (1996). Digital change detection in forest ecosystems with remote sensing imagery. Remote Sensing Reviews, 13, 207-234.

Coppin, P., Jonckheere, I., Nackaerts, K., Muys, B., \& Lambin, E. (2004). Review ArticleDigital change detection methods in ecosystem monitoring: a review. International Journal of Remote Sensing, 25, 1565-1596.

Crist, E. P., \& Cicone, R. C. (1984). A physically-based transformation of thematic mapper data - The TM tasseled cap. I.E.E.E. Transactions on Geoscience and Remote Sensing, GE-22, 256-263.

deBeurs, k. m, \& Henebry, G. M. (2006). Phenological Mixture Models: Using MODIS to identify key Phenological End Members and their spatial distribution in the northern Eurasian Semi-Arid Grain Belt. Eos AGU Fall Meeting Supplement, 87.

Eastman, J. R., \& Fulk, M. (1993). Long sequence time series evaluation using standardized principal components. Photogrammetric Engineering and Remote Sensing, 59, 1307-1312.

Elvidge, C. D., Ziskin, D., Baugh, K. E., Tuttle, B. T., \& Ghosh, T. (2009). A fifteen year record of global natural gas flaring derived from satellite data. Energies, 2, 595-622.

Fisher, J. I., \& Mustard, J. F. (2007). Cross-scalar satellite phenology from ground, Landsat, and MODIS data. Remote Sensing of Environment, 109, 261-273.

Fisher, J. I., Mustard, J. F., \& Vadeboncoeur, M. A. (2006). Green leaf phenology at Landsat resolution: Scaling from the field to the satellite. Remote Sensing of Environment, 100, 265-279.

Fung, T., \& LeDrew, E. (1987). Application of principal components analysis to change detection. Photogrammetric Engineering and Remote Sensing, 53, 1649-1658.

Galford, G. L., Mustard, J. F., Melillo, J., Gendrin, A., Cerri, C. C., \& Cerri, C. E. P. (2008). Wavelet analysis of MODIS time series to detect expansion and intensification of row-crop agriculture in Brazil. Remote Sensing of Environment, 112 576-587.

Gershenfeld, N. (1999). The nature of mathematical modeling. Cambridge England: Cambridge University Press.

Gillespie, A. R., Smith, M. O., Adams, J. B., Willis, S. C., Fischer, A. F., \& Sabol, D. E. (1990). Interpretation of residual images: spectral mixture analysis of AVIRIS images, Owens Valley, California. Proceedings of the 2nd Airborne Visible/Infrared Imaging Spectrometer (AVIRIS) Workshop (pp. 243-270). Pasadena, CA: NASA Jet Propulsion Laboratory.

Green, A. A., Berman, M., Switzer, P., \& Craig, M. D. (1988). A transformation for ordering mutispecral data in terms of image quality with implications for noise removal. I.E.E.E. Transactions on Geoscience and Remote Sensing, 26, 65-74.

Huete, A., Didan, K., Miura, T., Rodriguez, E. P., Gao, X., \& Ferreira, L. G. (2002). Overview of the radiometric and biophysical performance of the MODIS vegetation indices. Remote Sensing of Environment, 83, 195-213.

Johnson, P. E., Smith, M. O., \& Adams, J. B. (1985). Quantitative analysis of planetary reflectance spectra with principal components analysis. Journal of Geophysical Research, 90, C805-C810.

Kauth, R. J., \& Thomas, G. S. (1976). The Tasseled Cap - a graphic description of the spectral-temporal development of agricultural crops as seen by Landsat. Proceedings of the Symposium on Machine Porcessing of Remotely Sensed Data (pp. 4B41-44B51). West Lafayette, Indiana: Purdue University.

Lee, J. B., Woodyatt, A. S., \& Berman, M. (1990). Enhancement of high specral resoltuion remote sensing data by a noise-adjusted principal components tranform. I.E.E.E Transactions on Geoscience and Remote Sensing, 28, 295-304.

Lobell, D. B., \& Asner, G. P. (2004). Cropland distributions from temporal unmixing of MODIS data. Remote Sensing of Environment, 93, 412-422.

Lu, D., Mausel, P., Brondizio, E., \& Moran, E. (2004). Change detection techniques. International Journal of Remote Sensing, 25, 2365-2401.

Ozdogan, M. (2010). The spatial distribution of crop types from MODIS data: Temporal unmixing using Independent Component Analysis. Remote Sensing of Environment, 114, 1190-1204.

Parker, R. L. (1994). Geophysical Inverse Theory. Princeton, New Jersey: Princeton University Press.

Percival, D. B., \& Walden, A. T. (1993). Spectral analysis for physical applications. Cambridge, UK: Cambridge University Press.

Piwowar, J. M., Peddle, D. R., \& LeDrew, E. F. (1998). Temporal Mixture Analysis of Arctic sea ice imagery; A new approach for monitoring enviornmental change. Remote Sensing of Environment, 63, 195-207.

Piwowar, J. M., Peddle, D. R., \& Sauchyn, D. J. (2006). Identifying ecological variability in vegetation dynamics through temporal mixture analysis. Geoscience and Remote Sensing (IGARSS), IEEE International Symposium (pp. 3766-3770). : IEEE. 
Preisendorfer, R. W. (1988). Principal component analysis in meteorology and oceanography. Amsterdam: Elsevier.

Quarmby, N. A., Townshend, J. R. G., Settle, J. J., White, K. H., Milnes, M., Hindle, T. L. et al. (1992). Linear mixture modeling applied to AVHRR data for crop area estimation. International Journal of Remote Sensing, 13, 415-425.

Richards, J. A. (1984). Thematic mapping from multitemporal image data using the principal components transformation. Remote Sensing of Environment, 16, 35-46.

Settle, J. J., \& Drake, N. A. (1993). Linear mixing and the estimation of ground cover proportions. International Journal of Remote Sensing, 14, 1159-1177.

Singh, A., \& Harrison, A. (1985). Standardized Principal Components. International Journal of Remote Sensing, 6, 883-896.

Small, C. (2004). The landsat ETM plus spectral mixing space. Remote Sensing of Environment, 93, 1-17.

Small, C., \& Elvidge, C.D. (in press). Night on Earth: Mapping decadal changes of night light in Asia. International Journal of Applied Earth Observation and Geoinformation.

Small, C., \& Lu, J. W. T. (2006). Estimation and vicarious validation of urban vegetation abundance by spectral mixture analysis. Remote Sensing of Environment, 100, 441-456.

Smith, M. O., Johnson, P. E., \& Adams, J. B. (1985). Quantitative determination of mineral types and abundances from reflectance spectra using principal component analysis. Journal of Geophysical Research, 90, 792-804.
Smith, M. O., Ustin, S. L., Adams, J. B., \& Gillespie, A. R. (1990). Vegetation in deserts: I. A regional measure of abundance from multispectral images. Remote Sensing of Environment, 31, 1-26.

Tanre, D., Herman, M., Deschamps, P. Y., \& Leffe, A. D. (1979). Atmospheric Modeling For Space Measurements Of Ground Reflectances, Including Bidirectional Properties. Applied Optics, 18, 3587-3594.

Townshend, J. R. G., Goff, T. E., \& Tucker, C. J. (1985). Multitemporal dimensionality of images of normalized difference vegetation index at continental scales. I.E.E.E. Transactions on Geoscience and Remote Sensing, GE-23.

Tukey, J. W. (1977). Exploratory Data Analysis. : Addison-Wesley.

Verbesselt, J., Hyndman, R., Newnham, G., \& Culvenor, D. (2010). Detecting trend and seasonal changes in satellite image time series. Remote Sensing of Environment, 114, 106-115.

von Storch, H., \& Zwiers, F. W. (1999). Statistical Analysis in Climate Research. Cambridge UK: Cambridge University Press.

Zhang X., Friedl, M. A. \& Schaaf, C. B. (2006). Global vegetation phenology from Moderate Resolution Imaging Spectroradiometer (MODIS): Evaluation of global patterns and comparison with in situ measurements. Journal of Geophysical Research, $111,1-14$ 